TRANSACTIONS OF THE

AMERICAN MATHEMATICAL SOCIETY

Volume 365, Number 1, January 2013, Pages 23-58

S 0002-9947(2012)05468-7

Article electronically published on June 8, 2012

\title{
INFINITE DIMENSIONAL PERFECT SET THEOREMS
}

\author{
TAMÁS MÁTRAI
}

\begin{abstract}
What largeness and structural assumptions on $A \subseteq[\mathbb{R}]^{\omega}$ can guarantee the existence of a non-empty perfect set $P \subseteq \mathbb{R}$ such that $[P]^{\omega} \subseteq A$ ? Such a set $P$ is called $A$-homogeneous. We show that even if $A$ is open, in general it is independent of ZFC whether for a cardinal $\kappa$, the existence of an $A$-homogeneous set $H \in[\mathbb{R}]^{\kappa}$ implies the existence of a non-empty perfect $A$-homogeneous set.

On the other hand, we prove an infinite dimensional analogue of Mycielski's Theorem: if $A$ is large in the sense of a suitable Baire category-like notion, then there exists a non-empty perfect $A$-homogeneous set. We introduce fusion games to prove this and other infinite dimensional perfect set theorems.

Finally we apply this theory to show that it is independent of ZFC whether Tukey reductions of the maximal analytic cofinal type can be witnessed by definable Tukey maps.
\end{abstract}

\section{INTRODUCTION}

The Perfect Set Theorem says that an analytic subset of a Polish space is either countable or has a non-empty perfect subset (see e.g. [11, Theorem 29.1, p. 226]). The complexity assumption in this result is consistently optimal: in $L$ there exists an uncountable $\boldsymbol{\Pi}_{1}^{1}$ set without non-empty perfect subsets (see e.g. 9, Corollary 25.37]).

However, one is often obliged to quest for a perfect set which satisfies multidimensional relations. Let $N<\omega$ be fixed and let $[X]^{N}$ denote the set of $N$ element subsets of $X$. Then an $N$ dimensional perfect set theorem should address the following problem: what largeness and structural assumptions on $A \subseteq[X]^{N}$ can guarantee the existence of a non-empty perfect set $P \subseteq X$ such that $[P]^{N} \subseteq A$ ? Such a set $P$ is called $A$-homogeneous.

In [12, Theorem 2.2, p. 620], W. Kubiś proved that if $X$ is a Polish space, $A \subseteq[X]^{N}$ is $G_{\delta}$ and there exists an uncountable $A$-homogeneous set, then there exists a non-empty perfect $A$-homogeneous set. Obviously, as far as $G_{\delta}$ sets are concerned, this result is the exact multidimensional analogue of the Perfect Set Theorem. The surprising fact is that the complexity assumption in this result is also optimal: the Turing reducibility relation on $2^{\omega}$ (see Definition 2.4) defines an $F_{\sigma}$ set $A \subseteq\left[2^{\omega}\right]^{2}$ such that there exists an $A$-homogeneous set $H \subseteq 2^{\omega}$ with $|H|=\omega_{1}$ but there is no non-empty perfect $A$-homogeneous set.

For $A$ analytic, W. Kubiś and S. Shelah [13] investigated a rank function which decides whether the existence of an $A$-homogeneous set with a given cardinality implies the existence of a non-empty perfect $A$-homogeneous set (see also [8, [23]).

Received by the editors October 8, 2009 and, in revised form, September 20, 2010.

2010 Mathematics Subject Classification. Primary 03E15.

This research was partially supported by the OTKA grants K 61600, K 49786 and K 72655 and by the NSERC grants 129977 and A-7354.

(C) 2012 American Mathematical Society 23 
They obtain in particular that for every $\alpha<\omega_{1}$, it is consistent with ZFC that there exists an $F_{\sigma}$ set $A \subseteq\left[2^{\omega}\right]^{2}$ such that there exists an $A$-homogeneous set of cardinality $\aleph_{\alpha}$ but there is no non-empty perfect $A$-homogeneous set.

Nevertheless, the most frequently applied perfect set theorem is a classical result of J. Mycielski, which says that if $X$ is a non-empty perfect Polish space and $A_{N} \subseteq[X]^{N}(N<\omega)$ are co-meager relations, then there is a non-empty perfect set $P \subseteq X$ such that $[P]^{N} \subseteq A_{N}(N<\omega)$. So, in particular, if $A \subseteq[X]^{N}$ is co-meager, then there exists a non-empty perfect $A$-homogeneous set. Obviously, the largeness assumption in this result is not optimal.

In the present paper we study the existence of non-empty perfect homogeneous sets for infinite dimensional relations $A \subseteq[X]^{\omega}$. Unlike in the finite dimensional case, it is not obvious how to topologize $[X]^{\omega}$. Therefore we usually assume that $A \subseteq X^{\omega}$ is symmetric, i.e., it is invariant under permutations of coordinates in $X^{\omega}$ and a set $H \subseteq X$ is called $A$-homogeneous if the injective sequences of $H$, $I S_{\omega}(H)=\left\{\left(x_{n}\right)_{n<\omega} \in H^{\omega}: x_{n} \neq x_{m}(n<m<\omega)\right\}$, are in $A$.

It is obvious that if $A \subseteq X^{\omega}$ is closed and $H \in[X]^{\omega_{1}}$ is $A$-homogeneous, then $\mathrm{cl}_{X}(H)$ is also $A$-homogeneous. Hence there exists a non-empty perfect $A$ homogeneous set. In Section 2 we show that this complexity assumption is also optimal (see Theorem 2.1).

Theorem 1.1. Let $\kappa$ be a cardinal and suppose that there exists an $F_{\sigma}$ set $A \subseteq$ $\left[2^{\omega}\right]^{2}$ such that there exists an A-homogeneous set of cardinality $\kappa$ but there is no non-empty perfect $A$-homogeneous set. Then there exists a symmetric open set $U \subseteq\left(2^{\omega}\right)^{\omega}$ such that there exists a $U$-homogeneous set of cardinality $\kappa$ but there is no non-empty perfect $U$-homogeneous set.

Thus in the infinite dimensional case, by the above mentioned result of W. Kubiś and S. Shelah, even for open relations, it is consistent that the existence of a homogeneous set of large cardinality does not imply the existence of a non-empty perfect homogeneous set (see Corollary 2.3).

On the other hand, Mycielski's Theorem has an infinite dimensional analogue. For a Polish space $X$, consider the $\sigma$-ideal $\mathbb{M}$ generated by the sets of the form $\bigcup_{n<\omega}\left(M_{n} \times X^{\omega \backslash(n+1)}\right)$ where $M_{n} \subseteq X^{n+1}(n<\omega)$ are meager. As we will see, an easy application of Mycielski's Theorem yields that if $A \subseteq X^{\omega}$ satisfies $X^{\omega} \backslash A \in \mathbb{M}$, then there exists a non-empty perfect $A$-homogeneous set (see Theorem 4.1). A more involved task is to find sufficient conditions for $X^{\omega} \backslash A \in \mathbb{M}$. In Section 4 we provide such sufficient conditions. In particular, we will show the following (see Corollary 4.7/2).

Theorem 1.2. Let $X$ be a Polish space, let $A \subseteq X^{\omega}$ be co-analytic and suppose that there exists a non-meager A-homogeneous set. Then there exists a non-empty perfect A-homogeneous set.

As a corollary, we obtain that in the iterated perfect set model, for every coanalytic set $A \subseteq X^{\omega}$ if there exists an $A$-homogeneous set of cardinality continuum, then there exists a non-empty perfect $A$-homogeneous set (see Theorem 4.9). Moreover, we also obtain that in Cohen extensions the existence of a homogeneous set of sufficiently large cardinality implies the existence of a non-empty perfect homogeneous set (see Theorem 4.10). Thus by combining these results and Theorem 1.1, in Section 4 we will prove the following. 
Theorem 1.3. Let $1<\alpha<\omega_{1}$ be an ordinal. Then it is independent of ZFC whether for an open set $A \subseteq\left(2^{\omega}\right)^{\omega}$, the existence of an A-homogeneous set of cardinality $\aleph_{\alpha}$ implies the existence of a non-empty perfect $A$-homogeneous set.

The key result toward Theorem 1.2 is proved by using a game which is obtained as a fusion of Banach-Mazur games played on higher and higher dimensional powers of $X$ (see Definition 3.2). In Section 3 we introduce this game and characterize the winning strategies of the players (see Theorem 3.4). It seems that our procedure of taking fusions is applicable to a wide class of games of descriptive set theory. As an illustration, in Section 3.2 we briefly study the fusion of Perfect Set Property games (see Definition 3.13 and Corollary 3.18). Independent of our work, M. Sabok 22 introduced and studied similar games (see also 21]). We discuss the relation between fusion games and some games of 21], 22] and [30] in the introduction of Section 3 .

Our study of infinite dimensional perfect set theorems was motivated by the problem of whether Tukey reducibilities of the maximal analytic cofinal type $\mathcal{I}_{\max }$ can be witnessed by definable Tukey maps. In Section 5 we recall the relevant definitions and show that this problem is independent of ZFC (see Theorem 5.7).

Finally we would like to thank Pandelis Dodos, David H. Fremlin, Michael Hrušák, Arnold W. Miller, Benjamin D. Miller, Marcin Sabok, Lajos Soukup, Juris Steprāns, Boban Veličković, William A. R. Weiss and Jindřich Zapletal for their helpful discussions. We are indebted to Stevo Todorčević for his overall support in our research. We gratefully acknowledge the hospitality of the Department of Mathematics of Rutgers University, New Jersey.

\section{Open Relations}

The main result of this section is the following slightly generalized version of Theorem 1.1. Recall that for every set $H$ and $\alpha \leq \omega, I S_{\alpha}(H)=\left\{\left(x_{n}\right)_{n<\alpha} \in\right.$ $\left.H^{\alpha}: x_{n} \neq x_{m}(n<m<\alpha)\right\}$, and for $A \subseteq X^{\alpha}, H \subseteq X$ is called $A$-homogeneous if $I S_{\alpha}(H) \subseteq A$.

For every $\alpha \leq \omega$, let $S_{\alpha}$ denote the permutation group on $\alpha$. A set $A \subseteq X^{\alpha}$ is symmetric if for every $\pi \in S_{\alpha},\left(a_{k}\right)_{k<\alpha} \in A$ implies $\left(a_{\pi(k)}\right)_{k<\alpha} \in A$.

Theorem 2.1. Let $A \subseteq\left(2^{\omega}\right)^{2}$ be a symmetric $F_{\sigma}$ set such that there is no nonempty perfect $A$-homogeneous set. Then there is a symmetric open set $U \subseteq\left(2^{\omega}\right)^{\omega}$ such that

(1) for every $\left(x_{k}\right)_{k<\omega} \in\left(2^{\omega}\right)^{\omega}$, if $\left|\left\{x_{k}: k<\omega\right\}\right|=\omega$ and there are $i, j<\omega$ such that $x_{i} \neq x_{j}$ and $\left(x_{i}, x_{j}\right) \in A$, then $\left(x_{k}\right)_{k<\omega} \in U$;

(2) there is no non-empty perfect $U$-homogeneous set.

In particular, by 1, if $H \subseteq 2^{\omega}$ is A-homogeneous, then $H$ is $U$-homogeneous, as well.

Before proving Theorem 2.1, we need some preparation. A well-known theorem of F. Galvin states that if $X$ is a non-empty perfect Polish space and $B \subseteq[X]^{2}$ has Baire property, then either there is a non-empty perfect $B$-homogeneous set or there is a non-empty perfect $\left([X]^{2} \backslash B\right)$-homogeneous set (see e.g. [11, Theorem 19.7, p. 130]). We will use the following corollary.

Corollary 2.2. Let $A \subseteq\left(2^{\omega}\right)^{2}$ be a symmetric $F_{\sigma}$ set such that there is no nonempty perfect $A$-homogeneous set. Then every non-empty perfect set $P \subseteq 2^{\omega}$ has a non-empty perfect subset $Q \subseteq P$ such that $I S_{2}(Q) \cap A=\emptyset$. 
Proof. Apply Galvin's Theorem with $X=P$ and $B=A \cap[P]^{2}$.

We introduce some terminology and notation. Fix a metric $d_{1}$ on $2^{\omega}$. For every $0<n<\omega$, let $d_{n}$ denote the coordinate supremum metric on $\left(2^{\omega}\right)^{n}$ generated by $d_{1}$. With an abuse of notation, we extend $d_{n}$ to the hyperspace by setting, for every $A, B \subseteq\left(2^{\omega}\right)^{n}$,

$$
d_{n}(A, B)=\inf \left\{d_{n}(a, b): a \in A, b \in B\right\} .
$$

For every $0<n<\omega,\left(x_{k}\right)_{k<n} \in\left(2^{\omega}\right)^{n}$ and $\delta>0$, let

$$
B^{+}\left(\left(x_{k}\right)_{k<n}, \delta\right)=\left\{\left(y_{k}\right)_{k<\omega} \in\left(2^{\omega}\right)^{\omega}: \exists \pi \in S_{\omega}\left(d_{n}\left(\left(x_{k}\right)_{k<n},\left(y_{\pi(k)}\right)_{k<n}\right)<\delta\right)\right\} .
$$

Then the sets $B^{+}\left(\left(x_{k}\right)_{k<n}, \delta\right)$ are symmetric open subsets of $\left(2^{\omega}\right)^{\omega}$.

For every $1<n<\omega$ and $\left(x_{k}\right)_{k<n} \in\left(2^{\omega}\right)^{n}$, we define

$$
\delta\left(\left(x_{k}\right)_{k<n}\right)=\min \left\{\left|x_{i}-x_{j}\right|: i<j<n\right\} .
$$

For every $0<n<\omega, \Delta_{n}=\left\{(x, \ldots, x) \in\left(2^{\omega}\right)^{n}: x \in 2^{\omega}\right\}$.

Proof of Theorem 2.1, Let $F_{n} \subseteq\left(2^{\omega}\right)^{2}(n<\omega)$ be symmetric closed sets such that

$$
d_{2}\left(F_{n}, \Delta_{2}\right)>0(n<\omega) \text { and } A \backslash \Delta_{2}=\bigcup_{n<\omega} F_{n} .
$$

For every $n<\omega$, set

$$
U_{n}=\bigcup\left\{B^{+}\left(\left(x_{k}\right)_{k<n+3}, \delta\left(\left(x_{k}\right)_{k<n+3}\right) / 4\right):\left(x_{k}\right)_{k<n+3} \in\left(2^{\omega}\right)^{n+3},\left(x_{0}, x_{1}\right) \in F_{n}\right\} .
$$

We show that $U=\bigcup_{n<\omega} U_{n}$ fulfills the requirements.

By definition, $U$ is a symmetric open set. To see 1, let $\left(x_{k}\right)_{k<\omega} \in\left(2^{\omega}\right)^{\omega}$ be such that $\left|\left\{x_{k}: k<\omega\right\}\right|=\omega$ and there are $i, j<\omega$ such that $x_{i} \neq x_{j}$ and $\left(x_{i}, x_{j}\right) \in A$; say $\left(x_{i}, x_{j}\right) \in F_{n}$. Since $U$ is symmetric, to have $\left(x_{k}\right)_{k<\omega} \in U$ it is enough to show that $\left(x_{\pi(k)}\right)_{k<\omega} \in U$ for some $\pi \in S_{\omega}$. That is, we can assume $\left(x_{0}, x_{1}\right) \in F_{n}$ and $\left(x_{k}\right)_{k<n+3} \in I S_{n+3}\left(2^{\omega}\right)$. Then $\delta\left(\left(x_{k}\right)_{k<n+3}\right)>0$; hence $B^{+}\left(\left(x_{k}\right)_{k<n+3}, \delta\left(\left(x_{k}\right)_{k<n+3}\right) / 4\right) \neq \emptyset$. Thus $\left(x_{k}\right)_{k<\omega} \in U_{n} \subseteq U$, as required.

It remains to prove 2, Let $P \subseteq 2^{\omega}$ be an arbitrary non-empty perfect set. By Corollary 2.2. there is a non-empty perfect set $Q \subseteq P$ such that $I S_{2}(Q) \cap A=\emptyset$. We inductively define a sequence $\left(q_{k}\right)_{k<\omega} \in I S_{\omega}(Q)$ such that $\left(q_{k}\right)_{k<\omega} \notin U$; this will complete the proof. that

By induction on $n<\omega$, we define $q_{n} \in Q(n<\omega)$ and $\varepsilon_{n}>0(0<n<\omega)$ such

(i) for every $i, j<\omega, i \neq j$ implies $q_{i} \neq q_{j}$,

(ii) for every $0<n<\omega, \varepsilon_{n}=\min \left\{d_{2}\left(\left(q_{i}, q_{j}\right), F_{k}\right): i, j, k \leq n\right\}$,

(iii) for every $0<i<n<\omega, d_{1}\left(q_{n}, q_{i}\right)<\varepsilon_{i} / 2$.

Let $q_{0}, q_{1} \in Q$ be arbitrary satisfying $q_{0} \neq q_{1}$. Let $0<n<\omega$ and suppose that $q_{i}$ $(i \leq n)$ and $\varepsilon_{i}(0<i<n)$ are defined such that (ifliii) hold. In accordance with (iii), set

$$
\varepsilon_{n}=\min \left\{d_{2}\left(\left(q_{i}, q_{j}\right), F_{k}\right): i, j, k \leq n\right\} .
$$

Then $\varepsilon_{n}>0$ by $I S_{2}(Q) \cap A=\emptyset$ and (2.1).

To satisfy (ii) and (iii), let $q_{n+1} \in Q \backslash\left\{q_{i}: i \leq n\right\}$ be arbitrary satisfying $d_{1}\left(q_{n+1}, q_{i}\right)<\varepsilon_{i} / 2(i \leq n)$. By the inductive assumption (iii) for $n$, such a $q_{n+1}$ exists, namely any $q_{n+1} \in Q \backslash\left\{q_{i}: i \leq n\right\}$ sufficiently close to $q_{n}$ fulfills the requirements.

Suppose $\left(q_{k}\right)_{k<\omega} \in U$, say $\left(q_{k}\right)_{k<\omega} \in U_{n}$. Then $\left(q_{k}\right)_{k<\omega} \in B^{+}\left(\left(x_{k}\right)_{k<n+3}\right.$, $\left.\delta\left(\left(x_{k}\right)_{k<n+3}\right) / 4\right)$ for some $\left(x_{k}\right)_{k<n+3} \in\left(2^{\omega}\right)^{n+3}$ such that $\left(x_{0}, x_{1}\right) \in F_{n}$. Set $\delta=$ 
$\delta\left(\left(x_{k}\right)_{k<n+3}\right) / 4$. Then there are $k_{0}<k_{1}<\cdots<k_{n+2}<\omega$ and an enumeration $n+3=\left\{l_{i}: i<n+3\right\}$ such that

(iv) $d_{1}\left(q_{k_{i}}, x_{l_{i}}\right)<\delta / 4(i<n+3)$,

(v) for some $a<b<n+3,\left(x_{l_{a}}, x_{l_{b}}\right) \in F_{n}$.

We distinguish two cases. Suppose first that $b<n+2$. By (iv),

$$
d_{1}\left(q_{k_{a}}, x_{l_{a}}\right)<\frac{\delta}{4}, d_{1}\left(q_{k_{b}}, x_{l_{b}}\right)<\frac{\delta}{4} .
$$

We have $0<k_{n+1}<k_{n+2}$. So by (iii), $d_{1}\left(q_{k_{n+2}}, q_{k_{n+1}}\right)<\varepsilon_{k_{n+1}} / 2$. By $b<n+2$ we have $k_{b} \leq k_{n+1}$; in addition we have $n \leq k_{n+1}$, so $\varepsilon_{k_{n+1}} \leq d_{2}\left(\left(q_{k_{a}}, q_{k_{b}}\right), F_{n}\right)$. By (2.2) and (v) $), d_{2}\left(\left(q_{k_{a}}, q_{k_{b}}\right), F_{n}\right)<\delta / 4$. So to summarize,

$$
d_{1}\left(q_{k_{n+2}}, q_{k_{n+1}}\right)<\frac{\varepsilon_{k_{n+1}}}{2} \leq \frac{d_{2}\left(\left(q_{k_{a}}, q_{k_{b}}\right), F_{n}\right)}{2}<\frac{\delta}{8} .
$$

Again by (iv),

$$
d_{1}\left(q_{k_{n+1}}, x_{l_{n+1}}\right)<\frac{\delta}{4}, d_{1}\left(q_{k_{n+2}}, x_{l_{n+2}}\right)<\frac{\delta}{4},
$$

so the triangle inequality yields

$$
d_{1}\left(x_{l_{n+1}}, x_{l_{n+2}}\right) \leq d_{1}\left(x_{l_{n+1}}, q_{k_{n+1}}\right)+d_{1}\left(q_{k_{n+1}}, q_{k_{n+2}}\right)+d_{1}\left(q_{k_{n+2}}, x_{l_{n+2}}\right)<5 \frac{\delta}{8} .
$$

This contradicts the definition of $\delta$.

Finally suppose $b=n+2$; as in the previous case, we estimate $d_{1}\left(x_{l_{n+1}}, x_{l_{n+2}}\right)$. By (iv),

$$
d_{1}\left(q_{k_{a}}, x_{l_{a}}\right)<\frac{\delta}{4}, d_{1}\left(q_{k_{n+1}}, x_{l_{n+1}}\right)<\frac{\delta}{4}, d_{1}\left(q_{k_{n+2}}, x_{l_{n+2}}\right)<\frac{\delta}{4} .
$$

So by the triangle inequality,

$$
\begin{aligned}
d_{1}\left(x_{l_{n+1}}, x_{l_{n+2}}\right) & \leq d_{1}\left(x_{l_{n+1}}, q_{k_{n+1}}\right)+d_{1}\left(q_{k_{n+1}}, q_{k_{n+2}}\right)+d_{1}\left(q_{k_{n+2}}, x_{l_{n+2}}\right) \\
& <\frac{\delta}{2}+d_{1}\left(q_{k_{n+1}}, q_{k_{n+2}}\right) .
\end{aligned}
$$

We have $0<k_{n+1}<k_{n+2}$. So by (iii), $d_{1}\left(q_{k_{n+2}}, q_{k_{n+1}}\right)<\varepsilon_{k_{n+1}} / 2$. Since $k_{a} \leq k_{n+1}$ and $n \leq k_{n+1}$, we get $\varepsilon_{k_{n+1}} \leq d_{2}\left(\left(q_{k_{a}}, q_{k_{n+1}}\right), F_{n}\right)$. By (2.3),

$$
\begin{aligned}
d_{2}\left(\left(q_{k_{a}}, q_{k_{n+1}}\right), F_{n}\right) \leq d_{2}( & \left.\left(q_{k_{a}}, q_{k_{n+1}}\right),\left(x_{l_{a}}, x_{l_{n+2}}\right)\right) \\
& \leq \max \left\{\frac{\delta}{4}, d_{1}\left(q_{k_{n+1}}, x_{l_{n+2}}\right)\right\} \leq \frac{\delta}{4}+d_{1}\left(q_{k_{n+1}}, x_{l_{n+2}}\right) .
\end{aligned}
$$

By the triangle inequality and (2.3),

$$
d_{1}\left(q_{k_{n+1}}, x_{l_{n+2}}\right) \leq d_{1}\left(q_{k_{n+1}}, q_{k_{n+2}}\right)+d_{1}\left(q_{k_{n+2}}, x_{l_{n+2}}\right)<d_{1}\left(q_{k_{n+1}}, q_{k_{n+2}}\right)+\frac{\delta}{4} .
$$

To summarize,

$$
\begin{aligned}
d_{1}\left(q_{k_{n+2}}, q_{k_{n+1}}\right) & <\frac{\varepsilon_{k_{n+1}}}{2} \leq \frac{d_{2}\left(\left(q_{k_{a}}, q_{k_{n+1}}\right), F_{n}\right)}{2} \\
& \leq \frac{\delta}{8}+\frac{d_{1}\left(q_{k_{n+1}}, x_{l_{n+2}}\right)}{2}<\frac{\delta}{8}+\frac{d_{1}\left(q_{k_{n+1}}, q_{k_{n+2}}\right)}{2}+\frac{\delta}{8} ;
\end{aligned}
$$

i.e. $d_{1}\left(q_{k_{n+2}}, q_{k_{n+1}}\right)<\delta / 2$. By (2.4) we obtained $d_{1}\left(x_{l_{n+1}}, x_{l_{n+2}}\right)<\delta$, which again contradicts the definition of $\delta$. 
Corollary 2.3. For every $\alpha<\omega_{1}$, it is consistent with $Z F C$ that there exists a symmetric open set $U \subseteq\left(2^{\omega}\right)^{\omega}$ such that there exists a $U$-homogeneous set of cardinality $\aleph_{\alpha}$ but there is no non-empty perfect $U$-homogeneous set.

Proof. By [13, Corollary 5.13, p. 159] or [23, Theorem 1.13, p. 15], it is consistent with ZFC that there exists an $F_{\sigma}$ set $A \subseteq\left[2^{\omega}\right]^{2}$ such that there exists an $A$-homogeneous set of cardinality $\aleph_{\alpha}$ but there is no non-empty perfect $A$ homogeneous set. So the statement follows from Theorem 2.1.

As we mentioned in the introduction, the Turing reducibility relation is a ZFC example for an $F_{\sigma}$ set $T \subseteq\left[2^{\omega}\right]^{2}$ such that there exists an uncountable $T$-homogeneous set but there is no non-empty perfect $T$-homogeneous set. For the sake of completeness, we recall (a simplified version of) this relation and prove its above-mentioned properties.

Definition 2.4. For every $j<\omega$, set

$$
T_{j}^{<}=\left\{(x, y) \in 2^{\omega} \times 2^{\omega}: x(i)=y\left(2^{j+1} \cdot i+2^{j}\right)(i<\omega)\right\}
$$

and $T_{j}^{>}=\left\{(x, y) \in 2^{\omega} \times 2^{\omega}:(y, x) \in T_{j}^{<}\right\}$. Then the Turing reducibility relation $T \subseteq 2^{\omega} \times 2^{\omega}$ is defined by $T=\bigcup_{j<\omega}\left(T_{j}^{<} \cup T_{j}^{>}\right)$.

Proposition 2.5. The relation $T$ is symmetric and $F_{\sigma}$ is such that there exists an uncountable T-homogeneous set but there is no non-empty perfect T-homogeneous set.

Proof. It is obvious that $T_{j}^{<}, T_{j}^{>}(j<\omega)$ are closed sets, so $T$ is symmetric and $F_{\sigma}$. Observe that for given $\left(x_{j}\right)_{j<\omega} \subseteq 2^{\omega}$, the point $y \in 2^{\omega}$ defined by $y\left(2^{j+1} \cdot i+2^{j}\right)=$ $x_{j}(i)(i, j<\omega)$ satisfies $\left(x_{j}, y\right) \in T_{j}^{<} \subseteq T(j<\omega)$. Hence a straightforward transfinite recursion yields an uncountable $T$-homogeneous set.

Finally let $P \subseteq 2^{\omega}$ be a non-empty perfect set. Observe that for every $j<\omega$, $T_{j}^{>}$is the graph of a function; in particular, $T_{j}^{>} \cap(P \times P)(j<\omega)$ are meager in $P \times P$. By symmetry, this yields that $T \cap(P \times P)$ is meager in $P \times P$. Hence $P$ cannot be $T$-homogeneous, as required.

From $T$, by Theorem 2.1, we get a symmetric open set $U \subseteq\left(2^{\omega}\right)^{\omega}$ with analogous properties. In particular, this yields an example of an open set $U \subseteq\left(2^{\omega}\right)^{\omega}$ which is dense even in the box topology; still there is no non-empty perfect $U$-homogeneous set. We refer to Section 6.1 for a further discussion of alternative topologies.

\section{Fusion GAMES}

In this section we introduce the fusion of infinite sequences of games. The construction can be performed for most of the usual games of descriptive set theory. However, the method of the characterization of the winning strategies in a fusion game depends on the games whose fusion is taken. Therefore, here we study only the fusion game of Banach-Mazur games in detail, which is the most relevant for our perfect set theorems. In addition, in Section 3.2 we briefly discuss the fusion game of Perfect Set Property games.

Independent of our work, M. Sabok 22] introduced and studied games which are very similar to fusion games (see also 21]). The approach in these works, which originates from 30, makes explicit the connection between such games and iterated forcing. Fusion games and the corresponding ideal $\mathbb{M}$ (see Definition 3.3) are also to be compared to the games and ideals of [30, Definition 5.1.1, p. 225]. 
3.1. Banach-Mazur games. Let $X$ be a Choquet space such that there is a metric $d$ on $X$ whose balls are open in $X$. The diameter of a set $A \subseteq X$ is denoted by $\operatorname{diam}_{X}(A)$. Recall that for every $A \subseteq X$, in the Banach-Mazur game with payoff set $A$ (see e.g. [11, Section 21.D, p. 153]) two players play

$$
\begin{array}{llllllll}
I: & U(0) & & U(2) & & \ldots & U(2 n) & \\
& & & & & & & \\
I I: & & U(1) & & U(3) & \ldots & & U(2 n+1)
\end{array}
$$

where $U(n) \subseteq X(n<\omega)$ are non-empty open sets such that $U(n) \supseteq U(n+1)$ and $\operatorname{diam}_{X}(U(n))<2^{-n}(n<\omega)$, and player $I I$ wins the game if and only if $\bigcap_{i<\omega} U(i)$ is a singleton and $\bigcap_{i<\omega} U(i) \in A$.

The following is well known (see e.g. [11, Theorem 8.33, p. 51]).

Theorem 3.1. In the Banach-Mazur game with payoff set A,

(1) player I has a winning strategy if and only if there is a non-empty open set $U \subseteq X$ such that $A \cap U$ is meager;

(2) player II has a winning strategy if and only if $X \backslash A$ is meager.

We define the fusion game of Banach-Mazur games. If $Y$ is a set and $s, t \in Y^{<\omega}$, $|s|$ denotes the length of $s$ and $s \frown t$ stands for the sequence $s(0) \ldots$ $s(|s|-1) t(0) \ldots t(|t|-1)$. We write $s \sqsubseteq t$ if $s=\left.t\right|_{|s|}$. If $T \subseteq Y^{<\omega}$ is a tree and $n<\omega$, set $\operatorname{lev}_{n}(T)=\{t \in T:|t|=n\}$ and $[T]=\left\{\eta \in Y^{\omega}:\left.\eta\right|_{n} \in T(n<\omega)\right\}$. Also recall that the product space of Choquet spaces is Choquet (see e.g. [11, Exercise 8.13 , p. 44]).

Definition 3.2. For every $k<\omega$, let $X_{k}$ be a Choquet space such that there is a metric $d_{k}$ on $X_{k}$ whose balls are open in $X_{k}$. For every $A \subseteq \prod_{k<\omega} X_{k}, \mathcal{G}_{\omega}(A)$ denotes the fusion game of the Banach-Mazur games with payoff set $A$, in which players $I$ and $I I$ play

$$
\begin{aligned}
& I: U_{0}(0) \quad\left(\begin{array}{llll}
\left(U_{0}(2), U_{1}(0)\right) & \ldots & \left(U_{i}(2(n-i))\right)_{i \leq n} & \ldots
\end{array}\right. \\
& I I: \quad U_{0}(1) \quad\left(U_{0}(3), U_{1}(1)\right) \quad \ldots \quad\left(U_{i}(2(n-i)+1)\right)_{i \leq n} \quad \ldots
\end{aligned}
$$

where for every $k<\omega, U_{k}(i) \subseteq X_{k}(i<\omega)$ are non-empty open sets such that $U_{k}(i) \supseteq U_{k}(i+1)$ and $\operatorname{diam}_{X_{k}}\left(U_{k}(i)\right)<2^{-i}(i<\omega)$, and player $I I$ wins the game if and only if for every $k<\omega, \bigcap_{i<\omega} U_{k}(i)$ is a singleton and $\left(\bigcap_{i<\omega} U_{k}(i)\right)_{k<\omega} \in A$.

We denote by $\mathcal{G}_{\omega}$ the tree of partial runs of this game, ordered by end-extension. We set

$$
\mathcal{U}_{\omega}=\left\{\prod_{k<n} U_{k}: n<\omega, U_{k} \subseteq X_{k} \text { is non-empty open }(k<n)\right\} .
$$

A quasi-strategy of player $I$ is a non-empty pruned tree $\sigma \subseteq \mathcal{G}_{\omega}$ such that for every $n<\omega, U \in \operatorname{lev}_{2 n+1}(\sigma)$ and $u \in \mathcal{U}_{\omega}, U \smile u \in \mathcal{G}_{\omega}$ implies $U \smile u \in \sigma$.

Similarly, a quasi-strategy of player II is a non-empty pruned tree $\sigma \subseteq \mathcal{G}_{\omega}$ such that for every $n<\omega, U \in \operatorname{lev}_{2 n}(\sigma)$ and $u \in \mathcal{U}_{\omega}, U^{\frown} u \in \mathcal{G}_{\omega}$ implies $U^{\frown} u \in \sigma$.

For every pruned tree $\sigma \subseteq \mathcal{G}_{\omega}$, set

$$
\begin{array}{r}
W(\sigma)=\left\{\left(x_{k}\right)_{k<\omega} \in \prod_{k<\omega} X_{k}: \exists\left(\left(U_{i}(2(k-i))\right)_{i \leq k},\left(U_{i}(2(k-i)+1)\right)_{i \leq k}\right)_{k<w} \in[\sigma]\right. \\
\left.\left(x_{k}=\bigcap_{i<\omega} U_{k}(i)(k<\omega)\right)\right\} .
\end{array}
$$


For every $P \in\{I, I I\}, \Sigma(P)$ denotes the set of all quasi-strategies of player $P$ in the game $\mathcal{G}_{\omega}$, and we set $\mathcal{W}(P)=\left\{A \subseteq \prod_{k<\omega} X_{k}: \exists \sigma \in \Sigma(P)(W(\sigma) \subseteq A)\right\}$.

The winning strategies in $\mathcal{G}_{\omega}$ are characterized by the following Baire Categorylike notion. For arbitrary $A \subseteq X \times Y$, we set $\operatorname{Pr}_{X}(A)=\{x \in X: \exists y \in Y((x, y) \in$ A)\}.

Definition 3.3. With the notation of Definition 3.2. for an arbitrary sequence $S_{n} \subseteq \prod_{k \leq n} X_{k}(n<\omega)$ we set

$$
\left[\left(S_{n}\right)_{n<\omega}\right]=\bigcap_{n<\omega}\left(S_{n} \times \prod_{n<k<\omega} X_{k}\right) .
$$

We call $\mathbb{U}=\left(U_{n}\right)_{n<\omega}$ an open tower if for every $n<\omega, U_{n} \subseteq \prod_{k \leq n} X_{k}$ is an open set and $U_{n} \Delta \operatorname{Pr}_{\prod_{k \leq n} X_{k}}\left(U_{n+1}\right)$ is meager in $\prod_{k \leq n} X_{k}$. For two open towers $\mathbb{U}$ and $\mathbb{V}, \mathbb{U}$ is dense in $\mathbb{V}$ if $U_{n}$ is dense in $V_{n}(n<\omega)$ and $\mathbb{U}$ is dense if $U_{n}$ is dense in $\prod_{k \leq n} X_{k}(n<\omega)$. An open tower $\mathbb{U}$ is non-empty if $U_{0} \neq \emptyset$.

We define

$$
\begin{aligned}
\mathbb{M}=\{M \subseteq & \prod_{k<\omega} X_{k}: \exists M_{n} \subseteq \prod_{k \leq n} X_{k}(n<\omega) \\
& \left.\left(M_{n} \text { is meager in } \prod_{k \leq n} X_{k}(n<\omega), M \subseteq \bigcup_{n<\omega}\left(M_{n} \times \prod_{n<k<\omega} X_{k}\right)\right)\right\} .
\end{aligned}
$$

We discuss the relation of these notions to usual topologies before Lemma 3.7 . However, note that at this point it is not obvious that if $\mathbb{U}$ is a non-empty open tower, then $[\mathbb{U}] \neq \emptyset$. We will point out this corollary of $X_{k}(k<\omega)$ being Choquet after the proof of Proposition 3.9

We are in a position to state the characterization of the winning strategies in $\mathcal{G}_{\omega}$. Notice its analogy with Theorem 3.1

Theorem 3.4. With the notation of Definition 3.3 , for every $A \subseteq \prod_{k<\omega} X_{k}$

(1) the following are equivalent:

(a) $A \in \mathcal{W}(I)$;

(b) there is a non-empty open tower $\mathbb{U}$ such that $[\mathbb{U}] \subseteq A$;

(c) there is a non-empty open tower $\mathbb{U}$ such that $[\mathbb{U}] \backslash A \in \mathbb{M}$.

(2) the following are equivalent:

(a) $A \in \mathcal{W}(I I)$;

(b) there is a dense open tower $\mathbb{U}$ such that $[\mathbb{U}] \subseteq A$;

(c) $\left(\prod_{k<\omega} X_{k}\right) \backslash A \in \mathbb{M}$.

We will need that $\mathcal{G}_{\omega}(A)$ is determined for co-analytic $A$, as well. To this end, we introduce an unfolding of $\mathcal{G}_{\omega}$, as follows.

Definition 3.5. With the notation of Definition 3.2, for every $F \subseteq\left(\prod_{k<\omega} X_{k}\right) \times \omega^{\omega}$, $\mathcal{G}_{\omega}^{\star}(F)$ denotes the game with payoff set $F$ in which players $I$ and $I I$ play

$$
\begin{array}{lcrrrr}
I: U_{0}(0), y_{0} & \left(U_{0}(2), U_{1}(0)\right), y_{1} & \ldots & \left(U_{i}(2(n-i))\right)_{i \leq n}, y_{n} & \ldots \\
I I: & U_{0}(1) & \left(U_{0}(3), U_{1}(1)\right) & \ldots & \left(U_{i}(2(n-i)+1)\right)_{i \leq n} & \ldots
\end{array}
$$

where for every $n<\omega,\left(\left(U_{i}(2(k-i))\right)_{i \leq k},\left(U_{i}(2(k-i)+1)\right)_{i \leq k}\right)_{k \leq n} \in \mathcal{G}_{\omega}$ and $y_{n} \in \omega$, and player $I$ wins the game if and only if for every $k<\omega, \bigcap_{i<\omega} U_{k}(i)$ is a singleton 
and

$$
\left(\left(\bigcap_{i<\omega} U_{k}(i)\right)_{k<\omega},\left(y_{k}\right)_{k<\omega}\right) \in F
$$

For every pruned tree $\sigma \subseteq \mathcal{G}_{\omega}^{\star}$, set

$$
\begin{aligned}
& W(\sigma)=\left\{\left(\left(x_{k}\right)_{k<\omega},\left(y_{k}\right)_{k<\omega}\right) \in\left(\prod_{k<\omega} X_{k}\right) \times \omega^{\omega}:\right. \\
& \left.\exists\left(\left(U_{i}(2(k-i))\right)_{i \leq k}, y_{k},\left(U_{i}(2(k-i)+1)\right)_{i \leq k}\right)_{k<w} \in[\sigma]\left(x_{k}=\bigcap_{i<\omega} U_{k}(i)(k<\omega)\right)\right\} .
\end{aligned}
$$

For every $P \in\{I, I I\}, \Sigma^{\star}(P)$ denotes the set of all quasi-strategies of player $P$ in the game $\mathcal{G}_{\omega}^{\star}$. We set

$$
\begin{aligned}
\mathcal{W}^{\star}(I)=\left\{F \subseteq\left(\prod_{k<\omega} X_{k}\right) \times \omega^{\omega}: \exists \sigma \in \Sigma^{\star}(I)(W(\sigma) \subseteq F)\right\} \\
\\
\mathcal{W}^{\star}(I I)=\left\{F \subseteq\left(\prod_{k<\omega} X_{k}\right) \times \omega^{\omega}: \exists \sigma \in \Sigma^{\star}(I I)(W(\sigma) \cap F=\emptyset)\right\} .
\end{aligned}
$$

For the winning strategies in the game $\mathcal{G}_{\omega}^{\star}$ we have the following.

Theorem 3.6. With the notation of Definition 3.5, for every $F \subseteq\left(\prod_{k<\omega} X_{k}\right) \times \omega^{\omega}$,

(1) $F \in \mathcal{W}^{\star}(I)$ implies $\operatorname{Pr}_{\prod_{k<\omega} X_{k}}(F) \in \mathcal{W}(I)$;

(2) $F \in \mathcal{W}^{\star}(I I)$ implies $\operatorname{Pr}_{\prod_{k<\omega}} X_{k}(F) \in \mathbb{M}$.

It remains to prove our theorems. We start with the analysis of open towers vs. $\mathbb{M}$. It is obvious that every $M \in \mathbb{M}$ is meager in $\prod_{k<\omega} X_{k}$ even in the box topology. However, for an arbitrary non-empty open tower $\mathbb{U}$, [U్U] may also be nowhere dense in $\prod_{k<\omega} X_{k}$ in the box topology. Also, it is easy to construct two non-empty open towers $\mathbb{U}$ and $\mathbb{V}$ such that $[\mathbb{U}] \cap[\mathbb{V}]$ is a singleton, i.e., such an intersection may not contain a non-empty open tower. This indicates that open towers are not the open sets of a carefully chosen topology. As the next lemma shows, "meager" and "nowhere dense" coincide for M. In particular, once we obtained $[\mathbb{U}] \neq \emptyset$ for every non-empty open tower $\mathbb{U}$, from Corollary 3.8 we get $[\mathbb{U}] \notin \mathbb{M}$ for every non-empty open tower $\mathbb{U}$.

Lemma 3.7. With the notation of Definition 3.3, let $\mathbb{U}$ be a non-empty open tower and for every $n<\omega$, let $B_{n} \subseteq U_{n}$ be co-meager in $U_{n}$. Then there is a non-empty open tower $\mathbb{V}=\left(V_{n}\right)_{n<\omega}$ such that $\mathbb{V}$ is dense in $\mathbb{U}$ and $[\mathbb{V}] \subseteq\left[\left(B_{n}\right)_{n<\omega}\right]$.

Proof. For every $n<\omega$, let $R_{n}(i) \subseteq \prod_{k \leq n} X_{k}(i<\omega)$ be closed nowhere dense sets such that $U_{n} \backslash B_{n} \subseteq \bigcup_{i<\omega} R_{n}(i)$. Set

$$
V_{n}=U_{n} \backslash \bigcup_{i \leq n}\left(R_{n-i}(i) \times\left(\prod_{n-i<k \leq n} X_{k}\right)\right)(n<\omega) .
$$

It is obvious that $V_{n} \subseteq U_{n}$ is an open set which is dense in $U_{n}(n<\omega)$. We have $\left(x_{i}\right)_{i<\omega} \in[\mathbb{V}]$ if and only if $\left(x_{i}\right)_{i \leq n} \in V_{n}(n<\omega)$. So for every $n<\omega$, by $\left(x_{i}\right)_{i \leq n} \in V_{n}$ we have $\left(x_{i}\right)_{i \leq n} \in U_{n}$, and by $\left(x_{i}\right)_{i \leq n+k} \in V_{n+k}(k<\omega)$ we have $\left(x_{i}\right)_{i \leq n} \notin R_{n}(k)(k<\omega)$. Thus $\left(x_{i}\right)_{i \leq n} \in B_{n}(n<\omega)$, which completes the proof. 
Corollary 3.8. With the notation of Definition 3.3, let $A \subseteq \prod_{k<\omega} X_{k}$ be arbitrary and let $\mathbb{U}$ be a non-empty open tower. Then

(1) $[\mathbb{U}] \backslash A \in \mathbb{M}$ if and only if there is a non-empty open tower $\mathbb{V}$ such that $\mathbb{V}$ is dense in $\mathbb{U}$ and $[\mathbb{V}] \subseteq A$.

(2) $\left(\prod_{k<\omega} X_{k}\right) \backslash A \in \mathbb{M}$ if and only if there is a dense open tower $\mathbb{U}$ such that $[\mathbb{U}] \subseteq A$.

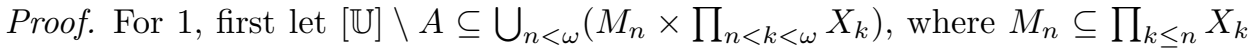
$(n<\omega)$ are meager. Then the statement follows from Lemma 3.7 applied with $B_{n}=U_{n} \backslash M_{n}(n<\omega)$. The converse is obvious.

Statement 2 is a special case of 1, so the proof is complete.

Proposition 3.9. With the notation of Definition 3.3, let $A \subseteq \prod_{k<\omega} X_{k}$ be arbitrary.

(1) If there is a non-empty open tower $\mathbb{U}$ such that $[\mathbb{U}] \subseteq A$, then $A \in \mathcal{W}(I)$.

(2) If there is a dense open tower $\mathbb{U}$ such that $[\mathbb{U}] \subseteq A$, then $A \in \mathcal{W}(I I)$.

Proof. Let $\mathbb{U}=\left(U_{n}\right)_{n<\omega}$. For 1, it is enough to construct a strategy $\sigma \in \Sigma(I)$ such that $W(\sigma) \subseteq[\mathbb{U}]$. We define $\sigma \subseteq \mathcal{G}_{\omega}$ by induction, as follows. Let $\left(U_{0}(0)\right) \in \sigma$ if and only if $\left(U_{0}(0)\right) \in \mathcal{G}_{\omega}$ and $U_{0}(0) \subseteq U_{0}$.

Let $n<\omega$ be arbitrary and suppose that $\sigma \cap \operatorname{lev}_{2 n+1}\left(\mathcal{G}_{\omega}\right)$ is already defined. For every $U \in \operatorname{lev}_{2 n+1}(\sigma), U_{i}(2(n-i)+1)(i \leq n)$ and $U_{i}(2(n+1-i))(i \leq n+1)$, let

$$
U \frown\left(\left(U_{i}(2(n-i)+1)\right)_{i \leq n}\right) \in \sigma
$$

and

$$
U \smile\left(\left(U_{i}(2(n-i)+1)\right)_{i \leq n},\left(U_{i}(2(n+1-i))\right)_{i \leq n+1}\right) \in \sigma
$$

if and only if

$$
U^{\frown}\left(\left(U_{i}(2(n-i)+1)\right)_{i \leq n},\left(U_{i}(2(n+1-i))\right)_{i \leq n+1}\right) \in \mathcal{G}_{\omega}
$$

and $\prod_{i \leq n+1} U_{i}(2(n+1-i)) \subseteq U_{n+1}$. This completes the inductive step of the definition of $\sigma$.

It is obvious that $\sigma \subseteq \mathcal{G}_{\omega}$ and that for every $n<\omega, U \in \operatorname{lev}_{2 n+1}(\sigma)$ and $u \in \mathcal{U}_{\omega}$, $U^{\frown} u \in \mathcal{G}_{\omega}$ implies $U^{-} u \in \sigma$. To see that $\sigma$ is pruned, let $n<\omega, U \in \operatorname{lev}_{2 n+1}(\sigma)$ and $\left(U_{i}(2(n-i)+1)\right)_{i \leq n}$ be arbitrary such that $U-\left(\left(U_{i}(2(n-i)+1)\right)_{i \leq n}\right) \in \sigma$. Since $\prod_{i \leq n} U_{i}(2(n-i)) \subseteq U_{n}$ and $U_{n} \Delta \operatorname{Pr}_{\prod_{k \leq n} X_{k}}\left(U_{n+1}\right)$ is meager,

$$
\left(\left(\prod_{i \leq n} U_{i}(2(n-i)+1)\right) \times X_{n+1}\right) \cap U_{n+1} \neq \emptyset .
$$

In particular, there are $U_{i}(2(n+1-i))(i \leq n+1)$ such that $U_{i}(2(n+1-i)) \subseteq$ $U_{i}(2(n-i)+1)(i \leq n), \operatorname{diam}_{X_{i}}\left(U_{i}(2(n+1-i))\right)<2^{-(n+1-i)}(i \leq n+1)$ and $\prod_{i \leq n+1} U_{i}(2(n+1-i)) \subseteq U_{n+1}$. Thus

$$
U^{-}\left(\left(U_{i}(2(n-i)+1)\right)_{i \leq n},\left(U_{i}(2(n+1-i))\right)_{i \leq n+1}\right) \in \sigma,
$$

which concludes $\sigma \in \Sigma(I)$.

It remains to prove $W(\sigma) \subseteq[\mathbb{U}]$. Let $\left(x_{k}\right)_{k<\omega} \in W(\sigma)$ be arbitrary, say $x_{k}=\bigcap_{i<\omega} U_{k}(i)(k<\omega)$ for some $U \in[\sigma]$. Then for every $n<\omega,\left(x_{k}\right)_{k \leq n} \in$ $\prod_{i \leq n} U_{i}(2(n-i)) \subseteq U_{n}$. This shows $\left(x_{k}\right)_{k<\omega} \in U_{n} \times\left(\prod_{n<k<\omega} X_{k}\right)(n<\omega)$, so the statement follows.

Statement 2 follows by an analogous argument. 
Since the spaces $X_{k}(k<\omega)$ are Choquet, in $\mathcal{G}_{\omega}$ both players can refine their quasi-strategies in such a way that the resulting sequence $\left(\left(U_{i}(2(k-i))\right)_{i \leq k}\right.$, $\left.\left(U_{i}(2(k-i)+1)\right)_{i \leq k}\right)_{k<\omega}$ satisfies $\bigcap_{i<\omega} U_{k}(i)$ is a singleton for every $k<\omega$. In particular, for every $P \in\{I, I I\}, A \in \mathcal{W}(P)$ implies $A \neq \emptyset$. So by Proposition 3.911, if $\mathbb{U}$ is a non-empty open tower, then $[\mathbb{U}] \neq \emptyset$.

The proof of the following proposition closely follows the proofs of [11, Theorem 8.33, p. 51] and [11, Theorem 21.8, p. 153].

Proposition 3.10. With the notation of Definition 3.3 and Definition 3.5,

(1) if $A \in \mathcal{W}(I)$, then there is a non-empty open tower $\mathbb{U}$ such that $[\mathbb{U}] \subseteq A$;

(2) if $A \in \mathcal{W}(I I)$, then there is a dense open tower $\mathbb{U}$ such that $[\mathbb{U}] \subseteq A$;

(3) if $F \in \mathcal{W}^{\star}(I I)$, then $\operatorname{Pr}_{\prod_{k<\omega} X_{k}}(F) \in \mathbb{M}$.

Proof. To see 1, let $\sigma \in \Sigma(I)$ be such that $W(\sigma) \subseteq A$. We define a tree $\tau \subseteq \sigma$ by induction, as follows. Let $\mathfrak{U}_{0} \subseteq \operatorname{lev}_{1}(\sigma)$ be a maximal family of pairwise disjoint open sets, and $\operatorname{set} \operatorname{lev}_{1}(\tau)=\mathfrak{U}_{0}$. Let $n<\omega$ and suppose that $\operatorname{lev}_{2 n+1}(\tau)$ is already defined. Let

$$
\mathfrak{U}_{n+1} \subseteq\left\{u \in \mathcal{U}_{\omega}: \exists U \in \operatorname{lev}_{2 n+1}(\tau) \exists v \in \mathcal{U}_{\omega}(U \frown(v, u) \in \sigma)\right\}
$$

be a maximal family of pairwise disjoint open sets. For every $u \in \mathfrak{U}_{n+1}$ fix one $v(u) \in \mathcal{U}_{\omega}$ such that $U \frown(v(u), u) \in \sigma$ and set

$$
\operatorname{lev}_{2 n+3}(\tau)=\left\{U \frown(v(u), u) \in \sigma: U \in \operatorname{lev}_{2 n+1}(\tau), u \in \mathfrak{U}_{n+1}\right\} .
$$

This completes the inductive step of the definition of $\tau$. Observe that by requiring the members of $\mathfrak{U}_{n}$ to be pairwise disjoint, for every $n<\omega$ and $u \in \mathfrak{U}_{n}$ there is a unique $U \in \operatorname{lev}_{2 n+1}(\tau)$ such that $U(2 n)=u$.

Let $U_{n}=\bigcup \mathfrak{U}_{n}(n<\omega)$; we show that $\mathbb{U}=\left(U_{n}\right)_{n<\omega}$ is a non-empty open tower and $[\mathbb{U}] \subseteq A$. It is obvious that $U_{0} \neq \emptyset$. Let $n<\omega$ be arbitrary and let $u \subseteq U_{n}$ be an arbitrary non-empty open set; we show $\left(\operatorname{Pr}_{\prod_{k \leq n} X_{k}}\left(U_{n+1}\right)\right) \cap u \neq \emptyset$. By passing to a subset we can assume $u \subseteq u^{\prime}$ for some $u^{\prime} \in \mathfrak{U}_{n}$. Then there is a unique $U \in \operatorname{lev}_{2 n+1}(\tau)$ such that $U(2 n)=u^{\prime}$. By the definition of $\mathcal{G}_{\omega}$, there is a $v \in \mathcal{U}_{\omega}$ such that $U \frown v \in \mathcal{G}_{\omega}$ and $v \subseteq u$. Since $\sigma$ is a strategy of player $I$, there is a $w \in \mathcal{U}_{\omega}$ such that $U \frown(v, w) \in \sigma$. In particular $\operatorname{Pr}_{\prod_{k \leq n} X_{k}}(w) \subseteq v$, hence $\operatorname{Pr}_{\prod_{k \leq n} X_{k}}(w) \subseteq u$. By the maximality of $\mathfrak{U}_{n+1}$, there is a $w^{\prime} \in \mathfrak{U}_{n+1}$ such that $w \cap w^{\prime} \neq \emptyset$. This shows $\left(\operatorname{Pr}_{\prod_{k \leq n} X_{k}}\left(U_{n+1}\right)\right) \cap u \neq \emptyset$. Since $u \subseteq U_{n}$ was arbitrary, we obtained $U_{n} \backslash \operatorname{Pr}_{\prod_{k \leq n} X_{k}}\left(U_{n+1}\right)$ is nowhere dense in $\prod_{k \leq n} X_{k}$. Since $\operatorname{Pr}_{\prod_{k \leq n} X_{k}}\left(U_{n+1}\right) \subseteq U_{n}$ follows immediately from the definition, we proved $U_{n} \Delta \operatorname{Pr}_{\prod_{k<n} X_{k}}\left(U_{n+1}\right)$ is meager in $\prod_{k \leq n} X_{k}(n<\omega)$. Thus $\mathbb{U}$ is a non-empty open tower. We also obtained that $\tau$ is a pruned tree. Since $\tau \subseteq \sigma$, we have $W(\tau) \subseteq W(\sigma) \subseteq A$, so it remains to see $[\mathbb{U}] \subseteq W(\tau)$.

Let $\left(x_{k}\right)_{k<\omega} \in[\mathbb{U}]$ be arbitrary. For every $n<\omega$, by $\mathfrak{U}_{n}$ being pairwise disjoint, there is a unique $v_{2 n} \in \mathfrak{U}_{n}$ such that $\left(x_{k}\right)_{k \leq n} \in v_{2 n}$. By the definition of $\tau$, $v_{2 n} \in \mathfrak{U}_{n}$ means that there is a $V_{n} \in \operatorname{lev}_{2 n+1}(\tau)$ with $V_{n}(2 n)=v_{2 n}$. But then $\left(x_{k}\right)_{k \leq i} \in V_{n}(2 i)$, which implies $V_{n}(2 i)=v_{2 i}(i<n)$. Thus there is a unique $V \in[\tau]$ such that $V(2 n)=v_{2 n}(n<\omega)$. This shows $\left(x_{k}\right)_{k<\omega} \in W(\tau)$, which completes the proof of 1 .

To see 2, let $\sigma \in \Sigma(I I)$ be such that $W(\sigma) \subseteq A$. As in the proof of 1 we can define maximal pairwise disjoint families $\mathfrak{U}_{n}$ of open subsets of $\prod_{k \leq n} X_{k}$ and a pruned tree $\tau \subseteq \sigma$ such that for every $n<\omega$ and $u \in \mathfrak{U}_{n}$ there is a unique 
$U \in \operatorname{lev}_{2 n+2}(\tau)$ satisfying $U(2 n+1)=u$. Set $U_{n}=\bigcup \mathfrak{U}_{n}(n<\omega)$. Since $\sigma$ is a strategy of player $I I$, the maximality of $\mathfrak{U}_{n}$ implies $U_{n}$ is dense in $\prod_{k \leq n} X_{k}$. Hence $\mathbb{U}=\left(U_{n}\right)_{n<\omega}$ is a dense open tower, and as in the proof of 1 , we have $[\mathbb{U}] \subseteq W(\tau) \subseteq W(\sigma) \subseteq A$.

To see 3, let $\sigma \in \Sigma^{\star}(I I)$ be such that $W(\sigma) \cap F=\emptyset$. For every $y \in \omega^{<\omega}$ we say $U \in \mathcal{G}_{\omega}$ is compatible with $\sigma, y$ if $|U|=2|y|$ and $(U(2 i), y(i), U(2 i+1))_{i<|y|} \in \sigma$.

For every $y \in \omega^{<\omega} \backslash\{\emptyset\}$ we construct a tree $\tau_{y} \subseteq \mathcal{G}_{\omega}$ of height $2|y|$ such that

(i) every $U \in \operatorname{lev}_{2|y|}\left(\tau_{y}\right)$ is compatible with $\sigma, y$;

(ii) $\left\{U(2|y|-1): U \in \operatorname{lev}_{2|y|}\left(\tau_{y}\right)\right\}$ is a family of pairwise disjoint open sets, and for every $U, V \in \operatorname{lev}_{2|y|}\left(\tau_{y}\right), U(2|y|-2) \neq V(2|y|-2)$ implies $U(2|y|-1) \cap$ $V(2|y|-1)=\emptyset$.

(iii) $y \sqsubseteq y^{\prime}$ implies $\tau_{y}$ is the restriction of $\tau_{y^{\prime}}$ to sequences of length $\leq 2|y|$;

(iv) $\tau_{y}$ is maximal with these properties.

Set $\tau_{\emptyset}=\emptyset$. Let $y \in \omega^{<\omega} \backslash\{\emptyset\}$ be arbitrary, set $y^{-}=\left.y\right|_{|y|-1}$ and suppose that $\tau_{y^{-}}$is already defined. Let

$$
\mathfrak{U}_{y} \subseteq\left\{u \in \mathcal{U}_{\omega}: \exists U \in \tau_{y^{-}} \exists v \in \mathcal{U}_{\omega}\left(U^{\frown}(v, u) \text { is compatible with } \sigma, y\right)\right\}
$$

be a maximal family of pairwise disjoint open sets. For every $u \in \mathfrak{U}_{y}$ fix one $v(u) \in \mathcal{U}_{\omega}$ such that $U^{-}(v(u), u)$ is compatible with $\sigma, y$ and set

$$
\operatorname{lev}_{2|y|}\left(\tau_{y}\right)=\left\{U \frown(v(u), u) \in \mathcal{G}_{\omega}: U \in \operatorname{lev}_{2|y|-2}\left(\tau_{y^{-}}\right), u \in \mathfrak{U}_{y}\right\} .
$$

This completes the inductive step of the definition of $\tau_{y}\left(y \in \omega^{<\omega} \backslash\{\emptyset\}\right)$.

It is obvious from the definition that (i)iv) hold. As in the proof of statement 2. for every $y \in \omega^{<\omega} \backslash\{\emptyset\}$ the maximality of $\mathfrak{U}_{y}$ implies $U_{y}=\bigcup \mathfrak{U}_{y}$ is dense in $\prod_{k<|y|} X_{k}$. Thus with

$$
M_{n}=\bigcup\left\{\left(\prod_{k<|y|} X_{k}\right) \backslash U_{y}: y \in \omega^{n+1}\right\},
$$

$M=\bigcup_{n<\omega}\left(M_{n} \times \prod_{n<k<\omega} X_{k}\right)$ satisfies $M \in \mathbb{M}$. It remains to show that $\operatorname{Pr}_{\prod_{k<\omega} X_{k}}(F)$ $\subseteq M$.

Let $\left(\left(x_{k}\right)_{k<\omega},\left(y_{k}\right)_{k<\omega}\right) \in\left(\prod_{k<\omega} X_{k}\right) \times \omega^{\omega}$ be arbitrary and suppose $\left(x_{k}\right)_{k<\omega} \notin$ $M$. Then in particular, $\left(x_{k}\right)_{k \leq n} \in U_{\left(y_{k}\right)_{k \leq n}}(n<\omega)$, so by (iii), there is a unique $U \in\left[\mathcal{G}_{\omega}\right]$ such that $\left.U\right|_{2 n+2} \in \tau_{\left(y_{k}\right)_{k \leq n}}(n<\omega)$. Then $\left(U(2 k), y_{k}, U(k+1)\right)_{k<\omega} \in$ $[\sigma]$, so $\left(\left(x_{k}\right)_{k<\omega},\left(y_{k}\right)_{k<\omega}\right) \in W(\sigma)$, which implies $\left(\left(x_{k}\right)_{k<\omega},\left(y_{k}\right)_{k<\omega}\right) \notin F$. This completes the proof.

Proof of Theorem [3.4, For 1, 1a $\Leftrightarrow 1 \mathrm{~b}$ follows from Proposition 3.911 and Proposition $3.10[1$, while $1 \mathrm{~b} \Leftrightarrow 1 \mathrm{c}$ is Corollary 3.811 , For 2 , $2 \mathrm{a} \Leftrightarrow \Leftrightarrow 2 \mathrm{~b}$ follows from Proposition 3.92 and Proposition 3.1022 and $2 \mathrm{~b} \Leftrightarrow 2 \mathrm{C}$ is Corollary 3.822 ,

Proof of Theorem 3.6. For 1 if $F \in \mathcal{W}^{\star}(I)$, then by omitting the $y_{i}$ s, player $I$ gets a strategy in $\mathcal{G}_{\omega}$ showing $\operatorname{Pr}_{\prod_{k<\omega} X_{k}}(F) \in \mathcal{W}(I)$. Statement 2 is Proposition 3.1013

The following is an immediate corollary of Theorem 3.4 and Theorem 3.6

Corollary 3.11. With the notation of Definition 3.2 and Definition 3.5, let $A \subseteq$ $\prod_{k<\omega} X_{k}$ and $F \subseteq\left(\prod_{k<\omega} X_{k}\right) \times \omega^{\omega}$ be arbitrary.

(1) If the game $\mathcal{G}_{\omega}(A)$ is determined, then either $\left(\prod_{k<\omega} X_{k}\right) \backslash A \in \mathbb{M}$ or there is a non-empty open tower $\mathbb{U}$ such that $A \cap[\mathbb{U}]=\emptyset$. 
(2) If the game $\mathcal{G}_{\omega}^{\star}(F)$ is determined, then either $\operatorname{Pr}_{\prod_{k<\omega} X_{k}}(F) \in \mathbb{M}$ or there is a non-empty open tower $\mathbb{U}$ such that $[\mathbb{U}] \subseteq \operatorname{Pr}_{\prod_{k<\omega} X_{k}}(F)$.

The following is to be compared to [21, Theorem 6.10], 22, Proposition 3.29, p. 31] and [30, Section 5.1.3, p. 231]. In Proposition 4.8 we will show that the complexity assumptions in this result are consistently optimal.

Corollary 3.12. With the notation of Definition 3.3,

(1) if $A \subseteq \prod_{k<\omega} X_{k}$ is an analytic set, then either $A \in \mathbb{M}$ or there is a nonempty open tower $\mathbb{U}$ such that $[\mathbb{U}] \subseteq A$.

(2) if $A \subseteq \prod_{k<\omega} X_{k}$ is a co-analytic set, then either $\left(\prod_{k<\omega} X_{k}\right) \backslash A \in \mathbb{M}$ or there is a non-empty open tower $\mathbb{U}$ such that $A \cap[\mathbb{U}]=\emptyset$; in particular, $\mathcal{G}_{\omega}(A)$ is determined.

Proof. For 1 let $F \subseteq\left(\prod_{k<\omega} X_{k}\right) \times \omega^{\omega}$ be a closed set such that $\operatorname{Pr}_{\prod_{k<\omega} X_{k}}(F)=A$. Then the game $\mathcal{G}_{\omega}^{\star}(F)$ is closed, hence determined, i.e., the statement follows from Corollary 3.112. Statement 2 follows from 1 by taking complements.

We note that Corollary 3.1211 has an alternative proof based on Borel determinacy, as follows. Since the $\sigma$-ideal $\mathbb{M}$ is generated by closed sets, by 24, Theorem 1, p. 1023] either $A \in \mathbb{M}$ or there is a $G_{\delta}$ set $G \subseteq A$ such that $G \notin \mathbb{M}$. The game $\mathcal{G}_{\omega}\left(\left(\prod_{k<\omega} X_{k}\right) \backslash G\right)$ is determined, so we conclude that there is a non-empty open tower $\mathbb{U}$ such that $[\mathbb{U}] \subseteq G \subseteq A$. However, we believe that by avoiding Borel determinacy and by presenting the unfolded game $\mathcal{G}_{\omega}^{\star}$ we give a better insight to the fusion game $\mathcal{G}_{\omega}$.

3.2. Perfect set property games. For simplicity, in this section we assume $X$ is a non-empty perfect Polish space and we fix a countable base $\mathcal{U}$ in $X$ which consists of non-empty open sets. We study the following games.

Definition 3.13. Let $0<N<\omega$ be fixed. For every $A \subseteq X^{N}, \mathcal{P}_{N}(A)$ denotes the $N$-dimensional Perfect Set Property game with payoff set $A$, in which two players play

$$
\begin{array}{lllll}
I:\left(\left(U_{k}(0,0), U_{k}(0,1)\right)\right)_{k<N} & & \ldots & \left(\left(U_{k}(n, 0), U_{k}(n, 1)\right)\right)_{k<N} & \ldots \\
I I: & \left(i_{k}(0)\right)_{k<N} & \ldots & \left(i_{k}(n)\right)_{k<N} & \ldots
\end{array}
$$

where for every $k<N, l<\omega$ and $j<2$ we have $i_{k}(l) \in\{0,1\}, U_{k}(l, j) \in \mathcal{U}$, $\operatorname{diam}_{X}\left(U_{k}(l, j)\right)<2^{-l}, U_{k}(l, 0) \cap U_{k}(l, 1)=\emptyset$ and $U_{k}(l+1, j) \subseteq U_{k}\left(l, i_{k}(l)\right)$. Player $I$ wins the game if and only if for every $k<N, \bigcap_{l<\omega} U_{k}\left(l, i_{k}(l)\right)$ is a singleton and $\left(\bigcap_{l<\omega} U_{k}\left(l, i_{k}(l)\right)\right)_{k<N} \in A$.

Similarly, for every $A \subseteq X^{\omega}, \mathcal{P}_{\omega}(A)$ denotes the fusion game of the Perfect Set Property games with payoff set $A$, in which two players play

$$
\begin{aligned}
& I:\left(U_{0}(0,0), U_{0}(0,1)\right) \quad\left(\left(U_{0}(1,0), U_{0}(1,1)\right),\left(U_{1}(0,0), U_{1}(0,1)\right)\right) \\
& I I: \quad i_{0}(0) \quad\left(i_{0}(1), i_{1}(0)\right) \\
& \left.\ldots \quad\left(U_{k}(n-k, 0), U_{k}(n-k, 1)\right)\right)_{k \leq n} \\
& \left(i_{k}(n-k)\right)_{k \leq n} \quad \ldots
\end{aligned}
$$

where for every $k, l<\omega$ and $j<2$ we have $i_{k}(l) \in\{0,1\}, U_{k}(l, j) \in \mathcal{U}, \operatorname{diam}_{X}\left(U_{k}(l, j)\right)$ $<2^{-(k+l)}, U_{k}(l, 0) \cap U_{k}(l, 1)=\emptyset$ and $U_{k}(l+1, j) \subseteq U_{k}\left(l, i_{k}(l)\right)$. Player $I$ wins 
the game if and only if for every $k<\omega, \bigcap_{l<\omega} U_{k}\left(l, i_{k}(l)\right)$ is a singleton and $\left(\bigcap_{l<\omega} U_{k}\left(l, i_{k}(l)\right)\right)_{k<\omega} \in A$.

Notice that $\mathcal{P}_{1}$ is the usual Perfect Set Property game (see e.g. [11, Section 21.A, p. 149]). The quasi-strategies of the players in the games $\mathcal{P}_{N}(0<N<\omega)$ and $\mathcal{P}_{\omega}$ are defined analogously to Definition 3.2. The characterization of existence of winning quasi-strategies involves the following notions. Recall $N_{s}=\left\{\sigma \in 2^{\omega}: s \sqsubseteq\right.$ $\sigma\}\left(s \in 2^{<\omega}\right)$.

Definition 3.14. Let $0<N<\omega$ be fixed. We call $\mathcal{C}=\left(\left(U_{k}(0), U_{k}(1)\right)\right)_{k<N}$ an $N$-cube if for every $k<N$ and $j<2$ we have $U_{k}(j) \in \mathcal{U}$ and $U_{k}(0) \cap U_{k}(1)=\emptyset$. We define $[\mathcal{C}]=\prod_{k<N}\left(U_{k}(0) \cup U_{k}(1)\right)$, and for every $t \in 2^{N}, \mathcal{C}(t)=\prod_{k<N} U_{k}(t(k))$. We set

$$
\operatorname{diam}_{X}(\mathcal{C})=\max \left\{\operatorname{diam}_{X}\left(U_{k}(j)\right): k<N, j<2\right\} .
$$

We say that $F \subseteq X^{N}$ is $N$-cube free if there is a $\delta>0$ such that for every $N$-cube $\mathcal{C}$ with $\operatorname{diam}_{X}(\mathcal{C})<\delta$ there is a $t \in 2^{N}$ with $F \cap \mathcal{C}(t)=\emptyset$. We set

$$
\begin{gathered}
\mathbb{F}_{N}=\left\{F \subseteq X^{N}: \exists F_{n} \subseteq X^{N}(n<\omega)\left(F_{n} \text { is } N \text {-cube free }(n<\omega), F \subseteq \bigcup_{n<\omega} F_{n}\right)\right\}, \\
\mathbb{F}_{\omega}=\left\{F \subseteq X^{\omega}: \exists F_{n} \subseteq X^{n+1}(n<\omega)\right. \\
\left.\quad\left(F_{n} \text { is }(n+1) \text {-cube free }(n<\omega), F \subseteq \bigcup_{n<\omega}\left(F_{n} \times X^{\omega \backslash(n+1)}\right)\right)\right\} .
\end{gathered}
$$

A function $f:\left(2^{\omega}\right)^{N} \rightarrow X^{N}$ is cube preserving if for every $n<\omega$ and $s_{k} \in 2^{n}$ $(k<N)$ there is an $N$-cube $\mathcal{C}$ such that $\operatorname{diam}_{X}(\mathcal{C})<2^{-n}$ and

$$
f\left[\prod_{k<N} N_{s_{\bar{k}}} t(k)\right]=f\left[\left(2^{\omega}\right)^{N}\right] \cap \mathcal{C}(t)\left(t \in 2^{N}\right) .
$$

A function $f:\left(2^{\omega}\right)^{\omega} \rightarrow X^{\omega}$ is cube preserving if for every $n<\omega$ and $s_{k} \in 2^{n}$ $(k \leq n)$ there is an $(n+1)$-cube $\mathcal{C}$ such that $\operatorname{diam}_{X}(\mathcal{C})<2^{-n}$ and

$$
f\left[\left(\prod_{k \leq n} N_{s_{\widehat{k}} t(k)}\right) \times X^{\omega \backslash(n+1)}\right]=f\left[\left(2^{\omega}\right)^{\omega}\right] \cap\left(\mathcal{C}(t) \times X^{\omega \backslash(n+1)}\right)\left(t \in 2^{n+1}\right) .
$$

Notice that $\mathbb{F}_{1}=[X]^{\leq \omega}$. It is easy to see that every cube preserving function is continuous and injective. The statement corresponding to Lemma 3.7 is the following.

Lemma 3.15. With the notation of Definition 3.14,

(1) $F \subseteq X^{N}$ is $N$-cube free if and only if $c l_{X^{N}}(F)$ is $N$-cube free.

(2) $\mathbb{F}_{N}(0<N<\omega)$ and $\mathbb{F}_{\omega}$ are $\sigma$-ideals generated by closed sets.

(3) $\mathbb{F}_{\omega}=\left\{F \subseteq X^{\omega}: \exists F_{n} \in \mathbb{F}_{n+1}(n<\omega)\left(F \subseteq \bigcup_{n<\omega}\left(F_{n} \times X^{\omega \backslash(n+1)}\right)\right)\right\}$.

Proof. Since $\mathcal{C}(t)$ is open for every $N$-cube $\mathcal{C}$ and $t \in 2^{N}$, the first statement follows. Then by definition, 2 holds for $\mathbb{F}_{N}(0<N<\omega)$ and $\mathbb{F}_{\omega}$ is generated by closed sets. To see 3 and that $\mathbb{F}_{\omega}$ is a $\sigma$-ideal, observe that if $F \subseteq X^{N}$ is $N$-cube free, then $F \times X^{M}$ is $N+M$-cube free $(M<\omega)$. So the statement follows by decomposing and re-indexing, as in the proof of Lemma 3.7 
Proposition 3.16. With the notation of Definition 3.13 and Definition 3.14,

(1) for every $A \subseteq X^{N}$, player $I$ has a winning quasi-strategy in $\mathcal{P}_{N}(A)$ if and only if there is a cube preserving function $f:\left(2^{\omega}\right)^{N} \rightarrow X^{N}$ such that $f\left[\left(2^{\omega}\right)^{N}\right] \subseteq A$;

(2) for every $A \subseteq X^{\omega}$, player $I$ has a winning quasi-strategy in $\mathcal{P}_{\omega}(A)$ if and only if there is a cube preserving function $f:\left(2^{\omega}\right)^{\omega} \rightarrow X^{\omega}$ such that $f\left[\left(2^{\omega}\right)^{\omega}\right] \subseteq A$.

Proof. For 1 suppose first that player $I$ has a winning quasi-strategy $\tau$. By passing to a non-empty pruned subtree we can assume that $\tau$ is a strategy, i.e., for every $n<\omega$ and $s \in \operatorname{lev}_{2 n}(\tau)$ there is a unique $N$-cube $\mathcal{C}$ with $s \frown \mathcal{C} \in \tau$. For every $n<\omega$ and $s_{k} \in 2^{n}(k<N)$ let $\mathcal{C}_{\left(s_{k}\right)_{k<N}}$ be an $N$-cube such that for every $\sigma_{k} \in 2^{\omega}$ $(k<N)$,

$$
\left(\mathcal{C}_{\left(\left.\sigma_{k}\right|_{m}\right)_{k<N}},\left(\sigma_{k}(m)\right)_{k<N}\right)_{m \leq n} \in \tau(n<\omega) ;
$$

this assignment is possible and unique since $\tau$ is a strategy. Moreover,

$$
\operatorname{diam}_{X}\left(\mathcal{C}_{\left(s_{k}\right)_{k<N}}\right)<2^{-n}\left(\left(s_{k}\right)_{k<N} \in\left(2^{n}\right)^{N}, n<\omega\right) .
$$

Define $f:\left(2^{\omega}\right)^{N} \rightarrow X^{N}$,

$$
f\left(\left(\sigma_{k}\right)_{k<N}\right)=\bigcap_{n<\omega} \mathcal{C}_{\left(\left.\sigma_{k}\right|_{n}\right)_{k<N}}
$$

Then the cubes $\mathcal{C}_{\left(s_{k}\right)_{k<N}}\left(\left(s_{k}\right)_{k<N} \in\left(2^{n}\right)^{N}, n<\omega\right)$ witness that $f$ is a cube preserving function. Since $\tau$ is a winning strategy of player $I, f\left[\left(2^{\omega}\right)^{N}\right] \subseteq A$ follows.

Now suppose there is a cube preserving function $f:\left(2^{\omega}\right)^{N} \rightarrow X^{N}$ such that $f\left[\left(2^{\omega}\right)^{N}\right] \subseteq A$, and let $\mathcal{C}_{\left(s_{k}\right)_{k<N}}\left(\left(s_{k}\right)_{k<N} \in\left(2^{n}\right)^{N}, n<\omega\right)$ be the witnessing cubes. By

$$
f\left[\left(2^{\omega}\right)^{N}\right] \cap\left[\mathcal{C}_{\left(s_{k}\right)_{k<N}}\right]=f\left[\prod_{k<N} N_{s_{k}}\right]=f\left[\left(2^{\omega}\right)^{N}\right] \cap \mathcal{C}_{\left(\left.s_{k}\right|_{n-1}\right)_{k<N}}\left(\left(s_{k}(n)\right)_{k<N}\right),
$$

we can assume

$$
\left[\mathcal{C}_{\left(s_{k}\right)_{k<N}}\right] \subseteq \mathcal{C}_{\left(\left.s_{k}\right|_{n-1}\right)_{k<N}}\left(\left(s_{k}(n)\right)_{k<N}\right)\left(\left(s_{k}\right)_{k<N} \in\left(2^{n}\right)^{N}, 0<n<\omega\right) .
$$

Then the non-empty pruned tree $\tau$ defined by (3.1) is a winning quasi-strategy for player $I$.

Statement 2 follows by an analogous argument.

Proposition 3.17. With the notation of Definition 3.13 and Definition 3.14 .

(1) for every $A \subseteq X^{N}$, player II has a winning quasi-strategy in $\mathcal{P}_{N}(A)$ if and only if $A \in \mathbb{F}_{N}$;

(2) for every $A \subseteq X^{\omega}$, player II has a winning quasi-strategy in $\mathcal{P}_{\omega}(A)$ if and only if $A \in \mathbb{F}_{\omega}$.

Proof. For 1 first suppose that player $I I$ has a winning quasi-strategy $\tau$. Let $U_{\emptyset}=X^{N}$. For every $0<n<\omega$ and $t \in \operatorname{lev}_{2 n}(\tau), \mathcal{C}_{t}=t(2 n-2)$ is an $N$-cube, so we can define $U_{t}=\mathcal{C}_{t}(t(2 n-1))$. Set

$$
F_{t}=U_{t} \backslash \bigcup\left\{\mathcal{C}(s): \mathcal{C} \text { is an } N \text {-cube, } s \in 2^{N}, t \frown \mathcal{C} \frown s \in \tau\right\}(t \in \tau) .
$$


We show $F_{t}(t \in \tau)$ are $N$-cube free. Fix $t \in \tau$ and let $\mathcal{C}=\left(\left(U_{k}(0), U_{k}(1)\right)\right)_{k<N}$ be an arbitrary $N$-cube with $\operatorname{diam}_{X}(\mathcal{C})<2^{-|t|}$; we find $s \in 2^{N}$ such that $F_{t} \cap \mathcal{C}(s)=\emptyset$. Since $U_{t}=\prod_{k<N} V_{k}$ for some $V_{k} \in \mathcal{U}(k<N)$, we have

$$
[\mathcal{C}] \cap U_{t}=\left[\left(\left(U_{k}(0) \cap V_{k}, U_{k}(1) \cap V_{k}\right)\right)_{k<N}\right] .
$$

So since $F_{t} \subseteq U_{t}$, we can assume $[\mathcal{C}] \subseteq U_{t}$. Then $t \frown \mathcal{C} \in \tau$, hence there is an $s \in 2^{N}$ with $t \frown \mathcal{C} \frown s \in \tau$. By definition, this implies $F_{t} \cap \mathcal{C}(s)=\emptyset$, as required.

Since $\tau$ is countable, it remains to show $A \subseteq \bigcup_{t \in \tau} F_{t}$. Suppose $\left(x_{k}\right)_{k<N} \in$ $X^{N} \backslash \bigcup_{t \in \tau} F_{t}$. By induction on $n$, we define $N$-cubes $\mathcal{C}_{n}$ and $s_{n} \in 2^{N}(n<\omega)$ such that $\left(\mathcal{C}_{n}, s_{n}\right)_{n<\omega} \in[\tau]$ and $\left(x_{k}\right)_{k<N} \in \bigcap_{n<\omega} \mathcal{C}_{n}\left(s_{n}\right)$. Since $\tau$ is a winning quasi-strategy of player $I I$, this implies $\left(x_{k}\right)_{k<N} \notin A$, as stated.

Since $\left(x_{k}\right)_{k<N} \notin F_{\emptyset}$, there are $\mathcal{C}_{0}, s_{0}$ with $\mathcal{C}_{0} \frown s_{0} \in \tau$ such that $\left(x_{k}\right)_{k<N} \in$ $\mathcal{C}_{0}\left(s_{0}\right)$. Let $n<\omega$ be arbitrary and suppose $\mathcal{C}_{i}, s_{i}, i \leq n$, are defined such that $\left(\mathcal{C}_{i}, s_{i}\right)_{i \leq n} \in \tau$ and $\left(x_{k}\right)_{k<N} \in \mathcal{C}_{n}\left(s_{n}\right)$. Since $\left(x_{k}\right)_{k<N} \in U_{\left(\mathcal{C}_{i}, s_{i}\right)_{i \leq n}} \backslash F_{\left(\mathcal{C}_{i}, s_{i}\right)_{i \leq n}}$, there are $\mathcal{C}_{n+1}, s_{n+1}$ with $\left(\mathcal{C}_{i}, s_{i}\right)_{i \leq n} \frown \mathcal{C}_{n+1} \frown s_{n+1} \in \tau$ and $\left(x_{k}\right)_{k<N} \in \mathcal{C}_{n+1}\left(s_{n+1}\right)$. This completes the inductive step of the definition of $\mathcal{C}_{n}, s_{n}(n<\omega)$, and the proof of 1 .

Statement 2 follows by an analogous argument.

Corollary 3.18. With the notation of Definition 3.13 and Definition 3.14 ,

(1) for every analytic set $A \subseteq X^{N}$, either $A \in \mathbb{F}_{N}$ or there is a cube preserving function $f:\left(2^{\omega}\right)^{N} \rightarrow X^{N}$ such that $f\left[\left(2^{\omega}\right)^{N}\right] \subseteq A$;

(2) for every analytic set $A \subseteq X^{\omega}$, either $A \in \mathbb{F}_{\omega}$ or there is a cube preserving function $f:\left(2^{\omega}\right)^{\omega} \rightarrow X^{\omega}$ such that $f\left[\left(2^{\omega}\right)^{\omega}\right] \subseteq A$.

Proof. To see 1 by Lemma 3.15ा2, the $\sigma$-ideal $\mathbb{F}_{N}$ is generated by closed sets. So by [24, Theorem 1, p. 1023], either $A \in \mathbb{F}_{N}$ or there is a $G_{\delta}$ set $G \subseteq A$ such that $G \notin \mathbb{F}_{N}$. The game $\mathcal{P}_{N}(G)$ is determined, so the statement follows from Proposition $3.16[1$ and Proposition 3.17]1.

Statement 2 follows by an analogous argument.

\section{Infinite Dimensional PERFECT SET theOREMS}

Our infinite dimensional perfect set theorems are based on the following easy observation. Recall that for every set $X$ and $\alpha \leq \omega, I S_{\alpha}(X)=\left\{\left(x_{n}\right)_{n<\alpha} \in\right.$ $\left.X^{\alpha}: x_{n} \neq x_{m}(n<m<\alpha)\right\}$.

Theorem 4.1. Let $X$ be a non-empty Choquet space such that $X$ has no isolated points and there is a metric $d$ on $X$ whose balls are open in $X$. Let $A \subseteq X^{\omega}$ satisfy $X^{\omega} \backslash A \in \mathbb{M}$. Then there is a non-empty perfect set $P \subseteq X$ such that $I S_{\omega}(P) \subseteq A$.

The proof of Theorem 4.1 uses the following version of Mycielski's Theorem (see e.g. [20, Theorem 1, p. 141] and [11, Exercise 19.5, p. 130]).

Theorem 4.2. Let $X$ be a non-empty Choquet space such that $X$ has no isolated points and there is a metric $d$ on $X$ whose balls are open in $X$. Let $M_{n} \subseteq X^{n+1}$ $(n<\omega)$ be meager sets. Then there is a non-empty perfect set $P \subseteq X$ such that for every $n<\omega$ we have $I S_{n+1}(P) \cap M_{n}=\emptyset$. 
Proof of Theorem 4.1. Let $M_{n} \subseteq X^{n+1}(n<\omega)$ be meager sets such that $X^{\omega} \backslash A \subseteq$ $\bigcup_{n<\omega}\left(M_{n} \times X^{\omega \backslash(n+1)}\right)$. By Theorem 4.2, there is a non-empty perfect set $P \subseteq X$ such that for every $n<\omega$ we have $I S_{n+1}(P) \cap M_{n}=\emptyset$. We show that $P$ fulfills the requirements.

If $\left(x_{i}\right)_{i<\omega} \in X^{\omega} \backslash A$, then there is an $n<\omega$ such that $\left(x_{i}\right)_{i<\omega} \in M_{n}$. Then $\left(x_{i}\right)_{i \leq n} \notin I S_{n+1}(P)$, hence $\left(x_{i}\right)_{i<\omega} \notin I S_{\omega}(P)$, as required.

Corollary 3.1222 say that for co-analytic $A, X^{\omega} \backslash A \in \mathbb{M}$ holds if there is no nonempty open tower $\mathbb{U}$ such that $[\mathbb{U}] \cap A=\emptyset$. In the sequel we give various sufficient conditions for this.

4.1. Largeness in category. Recall that for every $S_{n} \subseteq X^{n+1}(n<\omega)$ we have $\left[\left(S_{n}\right)_{n<\omega}\right]=\bigcap_{n<\omega}\left(S_{n} \times X^{\omega \backslash(n+1)}\right)$. If $n<\omega,\left(x_{i}\right)_{i \leq n} \in X^{n+1}$ and $S \subseteq X^{n+2}$, then we set

$$
[S]_{\left(x_{i}\right)_{i \leq n}}=\left\{x_{n+1} \in X:\left(x_{i}\right)_{i \leq n+1} \in S\right\} .
$$

The most important additional property our topological spaces have to satisfy is the following.

Definition 4.3. A topological space $X$ has the Kuratowski-Ulam property if for every $n<\omega$ and for every meager set $M \subseteq X^{n+2}$,

$$
\left\{\left(x_{i}\right)_{i \leq n} \in X^{n+1}:[M]_{\left(x_{i}\right)_{i \leq n}} \text { is non-meager in } X\right\}
$$

is meager in $X^{n+1}$.

By [11, Theorem 8.41, p. 53], every second countable topological space has the Kuratowski-Ulam property. In particular, all of our results hold for Polish spaces and for the canonical refinement of Polish topologies turning a countable family of analytic sets into clopen sets (see e.g. [11, Theorem 25.18, p. 203]).

Our main technical notion is the following.

Definition 4.4. Let $X$ be a topological space. We call $\mathbb{W}=\left(W_{n}\right)_{n<\omega}$ a flag if for every $n<\omega, W_{n} \subseteq X^{n+1}$ and $W_{n}=\operatorname{Pr}_{X^{n+1}}\left(W_{n+1}\right)$. A flag $\mathbb{W}$ is of second category everywhere if $W_{0} \subseteq X$ is of second category everywhere, and for every $n<\omega$ and $\left(x_{i}\right)_{i \leq n} \in W_{n}$ we have $\left[W_{n+1}\right]_{\left(x_{i}\right)_{i \leq n}}$ is of second category everywhere in $X$.

If $\mathbb{W}$ is a flag and $\mathbb{U}$ is an open tower, then we say $\mathbb{W}$ is co-meager in $\mathbb{U}$ if $U_{0} \backslash W_{0}$ is meager, and for every $n<\omega$ and $\left(x_{i}\right)_{i \leq n} \in W_{n} \cap U_{n}$ we have $\left[U_{n+1} \backslash W_{n+1}\right]_{\left(x_{i}\right)_{i \leq n}}$ is meager.

Lemma 4.5. Let $X$ be a topological space and let $\mathbb{U}$ be a non-empty open tower. If $X$ has the Kuratowski-Ulam property, then there is a flag $\mathbb{W}=\left(W_{n}\right)_{n<\omega}$ which is co-meager in $\mathbb{U}$ and $W_{n} \subseteq U_{n}(n<\omega)$. Moreover, if $X$ is Polish, then $W_{n}(n<\omega)$ can be taken $G_{\delta}$.

Proof. We define $T_{n}(i) \subseteq X^{n+1}(n, i<\omega)$ by induction, as follows. Set $T_{n}(0)=$ $U_{n} \Delta \operatorname{Pr}_{X^{n+1}}\left(U_{n+1}\right)(n<\omega)$. Let $i<\omega$ and suppose that $T_{n}(i)(n<\omega)$ are defined. Then let

$$
T_{n}(i+1)=\left\{\left(x_{i}\right)_{i \leq n} \in X^{n+1}:\left[T_{n+1}(i)\right]_{\left(x_{i}\right)_{i \leq n}} \text { is non-meager }\right\}(n<\omega) .
$$

We show that $W_{n}=U_{n} \backslash \bigcup_{i<\omega} \bigcup_{k \leq n}\left(T_{k}(i) \times X^{n-k}\right)(n<\omega)$ fulfill the requirements.

It is obvious that $W_{n} \subseteq U_{n}(n<\omega)$. Next we show that $U_{0} \backslash W_{0}$ is meager and that for every $n<\omega$ and $\left(x_{i}\right)_{i \leq n} \in W_{n}$ we have $\left[U_{n+1} \backslash W_{n+1}\right]_{\left(x_{i}\right)_{i \leq n}}$ is meager. Since $\mathbb{U}$ is an open tower, $T_{n}(0)(n<\omega)$ are meager. Using the Kuratowski-Ulam 
property of $X$, it is easy to see that for every $n, i<\omega, T_{n}(i) \subseteq X^{n+1}$ is meager. Hence $U_{0} \backslash W_{0}$ is meager, as required.

Now let $n<\omega$ and let $\left(x_{i}\right)_{i \leq n} \in W_{n}$. By $\left(x_{i}\right)_{i \leq n} \notin T_{n}(j+1)(j<\omega)$ we have $\left[T_{n+1}(j)\right]_{\left(x_{i}\right)_{i \leq n}}$ is meager $(j<\omega)$. So $\left[U_{n+1} \backslash W_{n+1}\right]_{\left(x_{i}\right)_{i \leq n}}$ is meager, as required.

To see that $\mathbb{W}$ is a flag, we have to show $W_{n}=\operatorname{Pr}_{X^{n+1}}\left(W_{n+1}\right)(n<\omega)$. Let first $n<\omega$ and $\left(x_{i}\right)_{i \leq n} \in W_{n}$ be arbitrary. By $\left(x_{i}\right)_{i \leq n} \notin T_{n}(0)$ we have $\left[U_{n+1}\right]_{\left(x_{i}\right)_{i \leq n}} \neq \emptyset$. As we have seen above, $\left[U_{n+1} \backslash W_{n+1}\right]_{\left(x_{i}\right)_{i \leq n}}$ is meager, in particular $\left[W_{n+1}\right]_{\left(x_{i}\right)_{i \leq n}} \neq \emptyset$, as required. If $n<\omega$ and $\left(x_{i}\right)_{i \leq n+1} \in W_{n+1}$ is arbitrary, then by $\left(x_{i}\right)_{i \leq n+1} \in U_{n+1}$ and $\left(x_{i}\right)_{i \leq n+1} \notin \bigcup_{i<\omega} \bigcup_{k \leq n+1}\left(T_{k}(i) \times X^{n+1-k}\right)$ we get $\left(x_{i}\right)_{i \leq n} \in U_{n}$ and $\left(x_{i}\right)_{i \leq n} \notin \bigcup_{i<\omega} \bigcup_{k \leq n}\left(T_{k}(i) \times X^{n-k}\right)$, i.e. $\left(x_{i}\right)_{i \leq n} \in W_{n}$, as required.

Finally, if $X$ is Polish, then $T_{n}(0)(n<\omega)$ are $F_{\sigma}$. By Montgomery's Theorem (see e.g. [11, Exercise 22.22, p. 174]), $T_{n}(i)(n, i<\omega)$ are $F_{\sigma}$, so by definition, $W_{n}$ $(n<\omega)$ are $G_{\delta}$.

Corollary 4.6. Let $X$ be a topological space with the Kuratowski-Ulam property, and let $\mathbb{U}$ be a non-empty open tower. Let $\mathbb{V}$ be flag which is of second category everywhere. Then $[\mathbb{U}] \cap[\mathbb{V}] \neq \emptyset$.

Proof. By Lemma 4.5, there is a flag $\mathbb{W}$ which is co-meager in $\mathbb{U}$ and $W_{n} \subseteq U_{n}$ $(n<\omega)$. By induction on $n<\omega$, we define $x_{n} \in X(n<\omega)$ such that $\left(x_{i}\right)_{i \leq n} \in$ $W_{n+1} \cap V_{n+1}(n<\omega)$. Then by $\left(x_{n}\right)_{n<\omega} \in[\mathbb{W}] \cap[\mathbb{V}]$ and $[\mathbb{W}] \subseteq[\mathbb{U}]$ the statement follows.

Since $U_{0} \neq \emptyset, U_{0} \backslash W_{0}$ is meager and $V_{0}$ is of second category everywhere, we have $W_{0} \cap V_{0} \neq \emptyset$. Let $x_{0} \in W_{0} \cap V_{0}$ be arbitrary.

Let $n<\omega$ and suppose that $x_{i}(i \leq n)$ are defined such that $\left(x_{i}\right)_{i \leq n} \in W_{n+1} \cap$ $V_{n+1}$. Since $\mathbb{W}$ is a flag, we get $\left[W_{n+2}\right]_{\left(x_{i}\right)_{i \leq n}} \neq \emptyset$. By $W_{n+2} \subseteq U_{n+2}$ this implies $\left[U_{n+2}\right]_{\left(x_{i}\right)_{i \leq n}} \neq \emptyset$. Moreover, $\left[U_{n+2} \backslash W_{n+2}\right]_{\left(x_{i}\right)_{i \leq n}}$ is meager, so since $\left[V_{n+2}\right]_{\left(x_{i}\right)_{i \leq n}}$ is of second category everywhere in $X$, we have $\left[V_{n+2}\right]_{\left(x_{i}\right)_{i \leq n}} \cap\left[W_{n+2}\right]_{\left(x_{i}\right)_{i \leq n}} \neq \emptyset$. Let $x_{n+1} \in\left[V_{n+2}\right]_{\left(x_{i}\right)_{i \leq n}} \cap\left[W_{n+2}\right]_{\left(x_{i}\right)_{i \leq n}}$ be arbitrary. This completes the inductive step of the construction and finishes the proof.

Corollary 4.7. Let $X$ be a non-empty Choquet space such that $X$ has no isolated points, $X$ has the Kuratowski-Ulam property and there is a metric $d$ on $X$ whose balls are open in $X$. Let $A \subseteq X^{\omega}$.

(1) Suppose $\mathcal{G}_{\omega}(A)$ is determined and there is an $H \subseteq X$ which is of second category everywhere and $I S_{\omega}(H) \subseteq A$. Then there exists a non-empty perfect set $P \subseteq X$ such that $I S_{\omega}(P) \subseteq A$.

(2) Suppose $A$ is co-analytic and there is an $H \subseteq X$ which is non-meager and $I S_{\omega}(H) \subseteq A$. Then there exists a non-empty perfect set $P \subseteq X$ such that $I S_{\omega}(P) \subseteq A$.

Proof. To see 1, set $V_{n}=\left\{\left(x_{i}\right)_{i \leq n} \in H^{n+1}: x_{i} \neq x_{j}(i<j \leq n)\right\}$; then $\mathbb{V}=$ $\left(V_{n}\right)_{n<\omega}$ is a flag which is of second category everywhere. Since $[\mathbb{V}] \subseteq A$, by Corollary 4.6 we have $A \cap[\mathbb{U}] \neq \emptyset$ for every non-empty open tower $\mathbb{U}$. Hence by Corollary 3.111], $X^{\omega} \backslash A \in \mathbb{M}$. So the statement follows from Theorem 4.1 .

By Corollary 3.1212, statement 2 reduces to 1 by passing to a non-empty open subset of $X$ where $H$ is of second category everywhere.

As we pointed out above, Corollary 4.7 is applicable if $X$ is Polish or $X$ is obtained from a Polish space by turning a countable family of analytic sets into 
clopen sets in the usual way. Even if largeness in the Baire category is not preserved during such refinement of topologies, this observation shows that the game $\mathcal{G}_{\omega}(A)$ is informative for $A=C \cap \prod_{k<\omega} A_{k}$, where $C \subseteq X$ is co-analytic and $A_{k} \subseteq X$ $(k<\omega)$ are analytic.

Finally we show that the complexity assumptions in Corollary 3.12 are consistently optimal. The assumption of the following proposition holds e.g. in $L$ (see e.g. [9, Corollary 25.28, p. 495]).

Proposition 4.8. Assume there exists a $\Sigma_{2}^{1}$ set $D \subseteq 2^{\omega}$ which does not have the Baire property. Then there exists a co-analytic set $A \subseteq\left(2^{\omega}\right)^{\omega}$ such that $A \notin \mathbb{M}$ but $[\mathbb{U}] \nsubseteq$ A for every non-empty open tower $\mathbb{U}$.

Proof. By passing to a relative open subset we can assume $D$ is non-meager and $2^{\omega} \backslash D$ is of second category everywhere. Let $C \subseteq 2^{\omega} \times 2^{\omega}$ be a co-analytic set such that projection of $C$ to the first coordinate is $D$. Let

$$
A=\left\{\left(x_{i}\right)_{i<\omega} \in\left(2^{\omega}\right)^{\omega}:\left(x_{0},\left(x_{i}(0)\right)_{0<i<\omega}\right) \in C\right\} .
$$

We show that $A$ fulfills the requirements.

To see that $A$ is co-analytic, let $\varphi: \omega \times \omega \rightarrow \omega \times \omega$ be a bijection defined by $\varphi(0, i)=(0, i)(i<\omega), \varphi(i, 0)=(1, i-1)(0<i<\omega)$ and $\varphi:\{(i, j) \in \omega \times \omega: i \cdot j \neq$ $0\} \rightarrow(\omega \backslash\{0,1\}) \times \omega$ being any bijection. Then the automorphism of $\left(2^{\omega}\right)^{\omega}$ induced by $\phi$ is a bijection between $A$ and $C \times\left(2^{\omega}\right)^{\omega} \backslash\{0,1\}$, hence $A$ is co-analytic.

Observe that the projection of $A$ to the first coordinate is $D$. If $\mathbb{U}$ is a nonempty open tower, then by Lemma 4.5, there exists a flag $\mathbb{W}=\left(W_{n}\right)_{n<\omega}$ which is co-meager in $\mathbb{U}$ and $W_{n} \subseteq U_{n}(n<\omega)$; in particular, $W_{0} \not \subset D$. Since the projection of $[\mathbb{W}]$ to the first coordinate is $W_{0}$, [WW] $\nsubseteq A$; hence $[\mathbb{U}] \nsubseteq A$, as required.

To see $A \notin \mathbb{M}$, let $M \in \mathbb{M}$ be arbitrary. By Corollary 3.812 , there is a dense open tower $\mathbb{U}$ satisfying $[\mathbb{U}] \cap M=\emptyset$. So by Lemma 4.5, there exists a flag $\mathbb{W}=\left(W_{n}\right)_{n<\omega}$ which is co-meager in $\mathbb{U}$ and $W_{n} \subseteq U_{n}(n<\omega)$; in particular, $W_{0}$ is co-meager in $2^{\omega}$ and $[\mathbb{W}] \cap M=\emptyset$.

Let $x_{0} \in W_{0} \cap D$ be arbitrary and let $x_{1} \in 2^{\omega}$ satisfy $\left(x_{0}, x_{1}\right) \in C$. Set

$$
U_{n}=\prod_{i \leq n}\left\{x \in 2^{\omega}: x(0)=x_{1}(i)\right\}(n<\omega)
$$

and $V_{n}=W_{n+1} \cap\left(\left\{x_{0}\right\} \times\left(2^{\omega}\right)^{n+1}\right)(n<\omega)$. Then $\mathbb{U}=\left(U_{n}\right)_{n<\omega}$ is a non-empty open tower and $\left\{x_{0}\right\} \times[\mathbb{U}] \subseteq A$, while $\mathbb{V}=\left(V_{n}\right)_{n<\omega}$ is a co-meager flag with $\left\{x_{0}\right\} \times[\mathbb{V}] \subseteq[\mathbb{W}]$. By Corollary 4.6 we have $[\mathbb{U}] \cap[\mathbb{V}] \neq \emptyset$, i.e. $A \cap[\mathbb{W}] \neq \emptyset$, and so $A \nsubseteq M$. This proves $A \notin \mathbb{M}$ and completes the proof.

4.2. Largeness in cardinality. We show that in the iterated perfect set model and in Cohen extensions the existence of a homogeneous set of sufficiently large cardinality implies the existence of a non-empty perfect homogeneous set.

Theorem 4.9. Let $V$ be a model obtained from a model of the Continuum Hypothesis by adding $\omega_{2}$ Sacks reals. Let $A \subseteq \mathbb{R}^{\omega}$ be a co-analytic set such that there is an A-homogeneous set of cardinality $\omega_{2}$. Then there exists a non-empty perfect A-homogeneous set.

Proof. Let $H \in[\mathbb{R}]^{\omega_{2}}$ be $A$-homogeneous. By [18, Theorem, p. 581], there is a nonempty perfect set $X \subseteq \mathbb{R}$ such that $H \cap X$ is of second category everywhere in $X$. Then the statement follows from Corollary 4.7/2 applied to $A \cap X^{\omega}$ and $H \cap X$. 
For every cardinal $\mu, C[\mu]=\{f \subseteq \mu \times \omega \rightarrow 2:|f|<\omega\}$ denotes the forcing for adding $\mu$ many Cohen reals. We will use the elementary properties of the Cohen forcing stated in [9, Lemma 26.4, p. 514], 14, Lemma 2.2, p. 250] and [14, Theorem 2.1, p. 252] without further reference.

Theorem 4.10. In $V$, let $\kappa=2^{\aleph_{0}}$ and let $\kappa<\lambda \leq \mu$ be arbitrary cardinals. In $V^{C[\mu]}$, let $A \subseteq\left(2^{\omega}\right)^{\omega}$ be a co-analytic set such that there is an A-homogeneous set of cardinality $\lambda$. Then there exists a non-empty perfect A-homogeneous set.

Proof. In $V^{C[\mu]}$, let $R=\left\{r_{\alpha}: \alpha<\mu\right\}$ be the $\mu$ many Cohen reals added to $V$. First suppose $H \in[R]^{\lambda}$, i.e., that there is an $I \in[\mu]^{\lambda}$ such that $H=\left\{r_{\alpha}: \alpha \in I\right\}$.

In $V^{C[\mu]}$, let $\mathcal{U}=\left\{U \subseteq 2^{\omega}: U\right.$ open, $\left.|U \cap H|<\lambda\right\}$. Set $U=\bigcup \mathcal{U}$. Then there is an $I_{U} \in[\mu]^{\leq \omega}$ such that $U \in V\left[\left\{r_{\alpha}: \alpha \in I_{U}\right\}\right]$. Since $\left\{r_{\alpha}: \alpha \notin I_{U}\right\}$ are Cohen reals over $V\left[\left\{r_{\alpha}: \alpha \in I_{U}\right\}\right], U$ cannot be dense in $2^{\omega}$. So we can find a non-empty open set $O \subseteq 2^{\omega} \backslash U$.

We show that in $V^{C[\mu]}$, every non-meager $G_{\delta}$ set $G \subseteq O$ satisfies $|G \cap H|=\lambda$. Given such a $G$, let $W \subseteq O$ be a non-empty open set such that $G$ is dense in $W$. Let $I_{G, W} \in[\mu] \leq \omega$ be such that $G, W \in V\left[\left\{r_{\alpha}: \alpha \in I_{G, W}\right\}\right]$. Since $W \subseteq O$, by the definition of $O$ we have $|H \cap W|=\lambda$. The set $H_{G, W}=\left\{r_{\alpha}: \alpha \in I_{G, W}\right\}$ is countable. So $H \cap W \backslash H_{G, W}$ has cardinality $\lambda$, and each member of this set is a Cohen real over $V\left[\left\{r_{\alpha}: \alpha \in I_{G, W}\right\}\right]$. Thus $H \cap W \backslash H_{G, W} \subseteq G$, so the statement follows.

By Theorem 4.1 in order to conclude the existence of a non-empty perfect $A$ homogeneous set, it is enough to show that $O^{\omega} \backslash A \in \mathbb{M}$. By Corollary 3.1212, this follows if we show that $A \cap[\mathbb{U}] \neq \emptyset$ for every non-empty open tower $\mathbb{U}=\left(U_{n}\right)_{n<\omega}$ with $U_{n} \subseteq O^{n+1}(n<\omega)$. So let $\mathbb{U}$ be such an open tower. By Lemma 4.5, there is a flag $\mathbb{W}=\left(W_{n}\right)_{n<\omega}$ which is co-meager in $\mathbb{U}$ and in which $W_{n}(n<\omega)$ are $G_{\delta}$. The proof will be complete if we show $A \cap[\mathbb{W}] \neq \emptyset$.

Let $I_{\mathbb{W}} \in[\mu]^{\leq \omega}$ be such that $\mathbb{W} \in V\left[\left\{r_{\alpha}: \alpha \in I_{\mathbb{W}}\right\}\right]$. We define a sequence $\left(h_{n}\right)_{n<\omega} \in I S_{\omega}(H) \cap[\mathbb{W}]$ by induction, as follows. Since $W_{0} \subseteq O$ is a non-meager $G_{\delta}$ set, we have $\left|H \cap W_{0}\right|=\lambda$. Let $h_{0} \in H \cap W_{0}$ be arbitrary.

Let $n<\omega$ be arbitrary and suppose that $h_{i}(i \leq n)$ are defined such that $\left(h_{i}\right)_{i \leq n} \in I S_{n+1}(H) \cap W_{n}$. By definition, $\left[W_{n+1}\right]_{\left(h_{i}\right)_{i<n}} \subseteq O$ is a non-meager $G_{\delta}$ set. So $\left|H \cap\left[W_{n+1}\right]_{\left(h_{i}\right)_{i<n}}\right|=\lambda$, thus we can pick $h_{n+1} \in H \cap\left[W_{n+1}\right]_{\left(h_{i}\right)_{i<n}} \backslash\left\{h_{i}: i \leq n\right\}$. This completes the inductive step of the definition of $\left(h_{n}\right)_{n<\omega}$ and completes the proof of the special $H \in[R]^{\lambda}$ case.

In the general case, let $H \in\left[2^{\omega}\right]^{\lambda}$ be $A$-homogeneous. For every $h \in H$ fix an $I_{h} \in[\mu] \leq \omega$ such that $h \in V\left[\left\{r_{\alpha}: \alpha \in I_{h}\right\}\right]$. By the standard $\Delta$-system argument and by extending $V$, we can assume $I_{h} \cap I_{h^{\prime}}=\emptyset\left(h, h^{\prime} \in H, h \neq h^{\prime}\right)$. By passing to a subset of $H$, we can assume in addition that $\operatorname{tp}\left(I_{h}\right)=\operatorname{tp}\left(I_{h^{\prime}}\right)=\eta<\omega_{1}\left(h, h^{\prime} \in H\right)$.

For every $h \in H$, let $f_{h}:\left(2^{\omega}\right)^{\eta} \rightarrow 2^{\omega}$ be a Borel function in $V$ such that $f_{h}\left(\left(r_{\alpha}\right)_{\alpha \in I_{h}}\right)=h$. By passing again to a subset of $H$, we can assume $f_{h}=f_{h^{\prime}}=f$ $\left(h, h^{\prime} \in H\right)$. Let $\mathfrak{X} \subseteq\left(2^{\omega}\right)^{\eta}$ be a co-meager $G_{\delta}$ set such that $\left.f\right|_{\mathfrak{X}}$ is continuous. Observe that for every $h \in H,\left(r_{\alpha}\right)_{\alpha \in I_{h}}$ is a Cohen real in $\left(2^{\omega}\right)^{\eta}$, so in particular $\left(r_{\alpha}\right)_{\alpha \in I_{h}} \in \mathfrak{X}(h \in H)$.

Set $\mathfrak{A}=\left\{\left(\mathfrak{x}_{k}\right)_{k<\omega} \in \mathfrak{X}^{\omega}:\left(f\left(\mathfrak{x}_{k}\right)\right)_{k<\omega} \in A \cap I S_{\omega}\left(2^{\omega}\right)\right\}$. As we observed above, $\mathfrak{H}=\left\{\left(r_{\alpha}\right)_{\alpha \in I_{h}}: h \in H\right\} \subseteq \mathfrak{X}$ is an $\mathfrak{A}$-homogeneous set of Cohen reals of cardinality $\lambda$. Since $\mathfrak{A}$ is co-analytic, by the special case of Theorem 4.10 proved above, there exists a non-empty perfect $\mathfrak{A}$-homogeneous set $\mathfrak{P} \subseteq \mathfrak{X}$.

Set $P=f[\mathfrak{P}]$. By definition, if $\left(\mathfrak{p}_{k}\right)_{k<\omega} \in I S_{\omega}(\mathfrak{P})$, then $\left(f\left(\mathfrak{p}_{k}\right)\right)_{k<\omega} \in I S_{\omega}\left(2^{\omega}\right)$. Hence $f$ is injective on $\mathfrak{P}$, i.e. by the continuity of $f, P$ is a non-empty perfect 
set. Similarly, for every $\left(\mathfrak{p}_{k}\right)_{k<\omega} \in I S_{\omega}(\mathfrak{P})$ we have $\left(f\left(\mathfrak{p}_{k}\right)\right)_{k<\omega} \in A$, so $P$ is $A$ homogeneous. This completes the proof.

Proof of Theorem 1.3. Let $1<\alpha<\omega_{1}$ be an ordinal. By [23, Claim 3.9, p. 39], it is consistent with ZFC that there exists an $F_{\sigma}$ set $C \subseteq\left[2^{\omega}\right]^{2}$ such that there exists a $C$-homogeneous set of cardinality $\aleph_{\alpha}$ but there is no non-empty perfect $C$-homogeneous set. Then by Theorem [1.1, there exists a symmetric open set $U \subseteq\left(2^{\omega}\right)^{\omega}$ such that there exists a $U$-homogeneous set of cardinality $\aleph_{\alpha}$ but there is no non-empty perfect $U$-homogeneous set.

On the other hand, by starting from a model with $2^{\aleph_{0}}=\aleph_{1}$ and by adding $\aleph_{\alpha+1}$ many Cohen reals, we get a model in which $2^{\aleph_{0}}=\aleph_{\alpha+1}$, and Theorem 4.10 implies the statement.

\section{An application: Definability of Tukey maps}

In this section a set is called bounded if it is bounded from above. Similarly, directed means upward directed. Let $(P, \leq)$ and $(Q, \leq)$ be directed partial orders. We say that $(P, \leq)$ is Tukey reducible to $(Q, \leq),(P, \leq) \leq_{T}(Q, \leq)$ in notation, if there is a function $f: P \rightarrow Q$ such that for every unbounded set $A \subseteq P, f[A] \subseteq Q$ is unbounded. Such an $f$ is called a Tukey map. If $(P, \leq) \leq_{T}(Q, \leq)$ and $(Q, \leq)$ $\leq_{T}(P, \leq)$, then $(P, \leq)$ and $(Q, \leq)$ are called Tukey equivalent, $(P, \leq) \equiv_{T}(Q, \leq)$ in notation. In the sequel we do not write out the partial order when it is obvious from the context.

An equivalent definition of Tukey reducibility is that $P \leq_{T} Q$ if and only if there is a function $g: Q \rightarrow P$ such that for every cofinal set $A \subseteq Q, g[A] \subseteq P$ is cofinal. This characterization indicates that Tukey reductions provide information about the cofinal types of directed partial orders and explains why Tukey equivalence classes are also called cofinal types.

Tukey reducibility turns out to be the right tool for the comparison of cofinal types. Not only does the existence of a Tukey reduction between two directed partial orders relate many of their structural properties (e.g. it is easy to see, using the equivalent definitions given above, that $P \leq_{T} Q$ implies add $(Q) \leq \operatorname{add}(P)$ and $\operatorname{cof}(P) \leq \operatorname{cof}(Q)$ ), but in addition, Tukey reductions account for many known inequalities between cardinal invariants (e.g. all inequalities in the Cichon diagram can be witnessed by Tukey maps; see e.g. [5] and [1]). So a natural question arises: how many cofinal types of directed partial orders are there?

The following result indicates that such a general endeavor has to face independence. As usual, for every cardinal $\kappa,[\kappa]^{<\omega}$ denotes the set of finite subsets of $\kappa$ partially ordered by inclusion. We remark that $[\kappa]^{<\omega}$ is the maximal cofinal type of directed partial orders of cardinality $\leq \kappa$ (see e.g. [29, Theorem 5.1, p. 13]).

Theorem 5.1 ([27, Theorem 9, p. 718]).

(1) If the Continuum Hypothesis holds, then there are $2^{\omega_{1}}$ many different cofinal types of directed partial orders of cardinality $\omega_{1}$.

(2) It is consistent with $Z F C$ that $\left\{1, \omega, \omega_{1}, \omega \times \omega_{1},\left[\omega_{1}\right]^{<\omega}\right\}$ are the only cofinal types of directed orders of cardinality $\leq \omega_{1}$.

Therefore it is reasonable to restrict the cofinal diversity problem to classes of directed orders which carry additional structures (see e.g. [5], [6]). One possible restriction is to assume definability properties. Accordingly, in the present section we study analytic ideals on $\omega$, i.e. such families $\mathcal{I} \subseteq \mathcal{P}(\omega)$ which form an ideal 
under the partial order $\subseteq$ and which are analytic subsets of $\mathcal{P}(\omega)$, endowed with the Cantor space topology. In Section 5.4 we will examine how restrictive this assumption is (see Proposition 5.25 and Proposition 5.26).

Recall that in the definition of Tukey reducibility the reducing functions are not required to possess any regularity properties. However, Tukey maps are not unique; e.g. if $f$ is a Tukey map and $f \leq f^{\prime}$ pointwise, then $f^{\prime}$ is also a Tukey map. So it is reasonable to ask whether a Tukey reduction between analytic ideals can be witnessed by "nice" Tukey maps (see [5, Problem 3N (c), p. 212], 16, Question 2, p. 193] and [28, Question 6.69] for analogous problems).

Problem 5.2. Let $\mathcal{I}, \mathcal{J} \subseteq \mathcal{P}(\omega)$ be analytic ideals satisfying $\mathcal{I} \leq_{T} \mathcal{J}$. Is there then a "definable" Tukey map $f: \mathcal{I} \rightarrow \mathcal{J}$ ?

Depending on $\mathcal{I}$ and $\mathcal{J}$, "definable" may mean continuous, Borel measurable, Souslin measurable (i.e. measurable with respect to the $\sigma$-algebra generated by analytic sets), Baire measurable, Lebesgue measurable, etc. An affirmative answer to this problem could allow the use of descriptive set theoretic methods for the study of an originally non-definable object.

Surprisingly, Problem 5.2 has an affirmative answer for many analytic ideals. In 25] the notion of basic directed partial orders was introduced and the following result was proved.

Theorem 5.3 ([25, Theorem 5.3, p. 1890]). Let $P, Q$ be basic directed partial orders satisfying $P \leq_{T} Q$. Then there is a Souslin measurable Tukey map $f: P \rightarrow Q$.

We will recall the definition of basic directed partial orders in Section 5.4 (see Definition 5.27). Here we only mention that every analytic $P$-ideal on $\omega$ is basic. However, there are many analytic ideals on $\omega$ which are not basic in any topology; we will call such ideals non-basic. In [16, Section 7, p. 190] a sequence of non-basic Borel ideals was constructed which is strictly decreasing in the Tukey hierarchy. In Section 5.4 we will prove the following.

Proposition 5.4. The structure $\left(\mathcal{P}(\omega), \subseteq^{\star}\right)$ embeds into the family of non-basic $F_{\sigma}$ ideals on $\omega$ partially ordered by $\leq_{T}$.

As we pointed out above, $\left[2^{\aleph_{0}}\right]<\omega$ is the maximal Tukey type among directed partial orders of cardinality $\leq 2^{\aleph_{0}}$, so in particular among analytic ideals. As we will recall in Section 5.4 this maximal cofinal type admits a representation as an $F_{\sigma}$ ideal on $\omega$.

Proposition 5.5 ([16, Proposition 3, p. 185]). There exists an $F_{\sigma}$ ideal $\mathcal{I}_{\max } \subseteq$ $\mathcal{P}(\omega)$ such that $\mathcal{I}_{\max } \equiv_{T}\left[2^{\aleph_{0}}\right]<\omega$

As we will see in Section 5.4, $\mathcal{I}_{\max }$ is not basic; in particular, Theorem 5.3 does not apply to its Tukey reductions. Therefore the following special case of Problem 5.2 is of particular interest (see e.g. [28, Question 6.69]).

Problem 5.6. Let $\mathcal{I} \subseteq \mathcal{P}(\omega)$ be an analytic ideal satisfying $\mathcal{I}_{\max } \leq_{T} \mathcal{I}$. Is there then a "definable" Tukey map $f: \mathcal{I}_{\max } \rightarrow \mathcal{I}$ ?

The purpose of this section is to show that even Problem [5.6 is independent of ZFC. 
Theorem 5.7. Let $\mathcal{I} \subseteq \mathcal{P}(\omega)$ be an arbitrary analytic ideal.

(1) Let $V$ be a model obtained from a model of the Continuum Hypothesis by adding $\omega_{2}$ Sacks reals. Then in $V$, if $\mathcal{I}_{\max } \leq_{T} \mathcal{I}$, then there is a continuous Tukey map $f: \mathcal{I}_{\max } \rightarrow \mathcal{I}$.

(2) If the Continuum Hypothesis holds, then there is an analytic ideal $\mathfrak{J} \subseteq \mathcal{P}(\omega)$ such that $\mathcal{I}_{\max } \leq_{T} \mathfrak{J}$, but if $f: \mathcal{I}_{\max } \rightarrow \mathfrak{J}$ is a Tukey map, then $f\left[\mathcal{I}_{\max }\right]$ has no non-empty perfect subsets. In particular, a Tukey map $f: \mathcal{I}_{\max } \rightarrow \mathfrak{J}$ cannot be Lebesgue measurable or have the Baire property.

As a corollary, in Section 5.2 we obtain that it is consistent with ZFC that $\mathcal{I}_{\max }$ has the primality property (see Definition 5.13 and Theorem [5.15).

Presently, we do not know about any other special cases of Problem 5.2 where the same independence phenomenon appears. What makes Tukey reductions of $\mathcal{I}_{\text {max }}$ particularly easy to describe is the following simple characterization.

Definition 5.8. Let $(P, \leq)$ be a directed partial order. A set $H \subseteq P$ is called strongly unbounded if every $A \in[H]^{\omega}$ is unbounded in $(P, \leq)$.

Proposition 5.9 ([16, Section 1, p. 174]). Let $(P, \leq)$ be a directed partial order and let $\kappa$ be an arbitrary cardinal. Then $[\kappa]^{<\omega} \leq_{T} P$ if and only if there exists a strongly unbounded set $H \in[P]^{\kappa}$.

5.1. A consistent positive answer to Problem 5.6. We will need the following simple observation.

Lemma 5.10. Let $P$ and $Q$ be directed partial orders and let $f: P \rightarrow Q$ be a Tukey map. If $H \subseteq P$ is strongly unbounded, then $\left.f\right|_{H}$ is finite-to-one and $f[H] \subseteq Q$ is also strongly unbounded.

Proof. By definition, every $A \in[H]^{\omega}$ is unbounded. Since $f$ is a Tukey map, $f[A]$ cannot be a singleton, i.e., $f$ is finite-to-one. The second statement immediately follows from the definition.

The following implies Theorem 5.7/1 and also shows that the conclusion of Theorem 5.711 holds for every projective ideal under suitable large cardinal assumptions.

Theorem 5.11. Let $\boldsymbol{\Gamma}$ be a projective pointclass such that every $I \in \boldsymbol{\Gamma}(\mathcal{P}(\omega))$ has the Baire property, and for every $A \in \boldsymbol{\Gamma}\left(\mathcal{P}(\omega)^{\omega}\right)$ the game $\mathcal{G}_{\omega}(A)$ is determined. Let $\kappa \leq 2^{\aleph_{0}}$ be a cardinal such that for every $H \in[\mathcal{P}(\omega)]^{\kappa}$ there exists a non-empty perfect set $Q \subseteq \mathcal{P}(\omega)$ such that $H \cap Q$ is of second category everywhere in $Q$. Let $I \subseteq \mathcal{P}(\omega)$ be an ideal with $\mathcal{P}(\omega) \backslash I \in \boldsymbol{\Gamma}(\mathcal{P}(\omega))$ such that $[\kappa]^{<\omega} \leq_{T} I$. Then there is a continuous Tukey reduction from $\left[2^{\omega}\right]^{<\omega}$ to $I$.

Proof. Let $f:[\kappa]^{<\omega} \rightarrow I$ be a Tukey map and let $H=f[\kappa]$. By Lemma 5.10, $f$ is finite-to-one, hence $H$ has cardinality $\kappa$. So there exists a non-empty perfect set $Q \subseteq \mathcal{P}(\omega)$ such that $H \cap Q$ is of second category everywhere in $Q$. Since $I \cap Q$ has the Baire property in $Q$, by $H \cap Q \subseteq I \cap Q$ there is a co-meager $G_{\delta}$ set $X \subseteq I \cap Q \subseteq \mathcal{P}(\omega)$ satisfying $H \cap X$ is of second category everywhere in $X$.

Set $A=\left\{\left(x_{i}\right)_{i<\omega} \in X^{\omega}: \bigcup_{i<\omega} x_{i} \notin I\right\}$. Since the union function from $\mathcal{P}(\omega)^{\omega}$ to $\mathcal{P}(\omega)$ is Borel and $\mathcal{P}(\omega) \backslash I \in \boldsymbol{\Gamma}(\mathcal{P}(\omega))$, we have $A \in \boldsymbol{\Gamma}\left(X^{\omega}\right)$. Then the game $\mathcal{G}_{\omega}(A)$ is determined and $I S_{\omega}(H \cap X) \subseteq A$, so by Corollary 4.7/1 there is a nonempty perfect set $P \subseteq X$ such that $I S_{\omega}(P) \subseteq A$. Then any continuous bijection $\tilde{f}: 2^{\omega} \rightarrow P$ is a continuous Tukey map from $\left[2^{\omega}\right]^{<\omega}$ to $I$. 
Proof of Theorem [5.711. Recall that $\mathcal{I}_{\max } \equiv_{T}\left[2^{\omega}\right]^{<\omega}$. By [18, Theorem, p. 581], in the iterated perfect set model for every $H \in\left[2^{\omega}\right] 2^{\aleph_{0}}$ there exists a non-empty perfect set $Q \subseteq \mathcal{P}(\omega)$ such that $H \cap Q$ is of second category everywhere in $Q$. So by Corollary [3.1222, the statement follows from Theorem [5.11 with $\Gamma=\Pi_{1}^{1}$ and $\kappa=2^{\aleph_{0}}=\omega_{2}$.

5.2. Consistent primality of $\mathcal{I}_{\max }$. It is easy to see that the least upper bound in the Tukey order of two ideals $\mathcal{I}, \mathcal{J} \subseteq \mathcal{P}(\omega)$ is their direct sum, or disjoint union, defined as follows.

Definition 5.12. Let $\mathcal{I}, \mathcal{J} \subseteq \mathcal{P}(\omega)$ be arbitrary ideals. Let $E=\{2 n: n<\omega\}$. Then $\mathcal{I} \oplus \mathcal{J} \subseteq \mathcal{P}(\omega)$ is the ideal defined by

$$
A \in \mathcal{I} \oplus \mathcal{J} \Leftrightarrow\{n<\omega: 2 n \in A \cap E\} \in \mathcal{I} \text { and }\{n<\omega: 2 n+1 \in A \backslash E\} \in \mathcal{J} .
$$

For a complete description of the cofinal types of analytic ideals, ideals having the following primality property are of particular importance.

Definition 5.13. We say that an ideal $\mathcal{I} \subseteq \mathcal{P}(\omega)$ has the primality property if for every ideal $\mathcal{J}, \mathcal{K} \subseteq \mathcal{P}(\omega), \mathcal{I} \leq_{T} \mathcal{J} \oplus \mathcal{K}$ implies $\mathcal{I} \leq_{T} \mathcal{J}$ or $\mathcal{I} \leq_{T} \mathcal{K}$.

It is reasonable to ask which ideals have the primality property, and especially whether $\mathcal{I}_{\max }$ has the primality property (see e.g. [28, Question 6.68]). Note that by [27, Theorem 6, p. 715] the primality of $\mathcal{I}_{\max }$ fails among non-definable ideals. On the other hand, by [28. Theorem 6.71], the primality of $\mathcal{I}_{\max }$ holds for Souslin measurable Tukey reductions, as follows.

Theorem 5.14 ([28, Theorem 6.71]). Let $\mathcal{I}, \mathcal{J} \subseteq \mathcal{P}(\omega)$ be analytic ideals such that there exists a Souslin-measurable Tukey reduction of $\mathcal{I}_{\max }$ to $\mathcal{I} \oplus \mathcal{J}$. Then either $\mathcal{I}_{\max } \leq_{T} \mathcal{I}$ or $\mathcal{I}_{\max } \leq_{T} \mathcal{J}$.

So by Theorem 5.711, we get the following.

Theorem 5.15. Let $V$ be a model obtained from a model of the Continuum Hypothesis by adding $\omega_{2}$ Sacks reals. In $V$, let $\mathcal{I}, \mathcal{J} \subseteq \mathcal{P}(\omega)$ be analytic ideals such that $\mathcal{I}_{\max } \leq_{T} \mathcal{I} \oplus \mathcal{J}$. Then either $\mathcal{I}_{\max } \leq_{T} \mathcal{I}$ or $\mathcal{I}_{\max } \leq_{T} \mathcal{J}$.

Proof. By Theorem 5.711, there exists a continuous Tukey reduction of $\mathcal{I}_{\max }$ to $\mathcal{I} \oplus \mathcal{J}$. So the statement follows from Theorem 5.14,

5.3. A consistent negative answer to Problem 5.6. In this section we construct the ideal $\mathfrak{J}$ of Theorem 5.7/2. To this end, the main technical step is to observe that it is enough to construct an ideal of compact sets with the same properties.

Definition 5.16. Let $\mathcal{K}\left(2^{\omega}\right)$ denote the space of compact subsets of the Cantor set endowed with the Vietoris topology. A family $\mathcal{I} \subseteq \mathcal{K}\left(2^{\omega}\right)$ is called an ideal of compact sets if $\mathcal{I}$ is closed under taking finite unions and $\mathcal{I}$ is closed downward, i.e. for every $K, L \in \mathcal{K}\left(2^{\omega}\right), K \subseteq L \in \mathcal{I}$ implies $K \in \mathcal{I}$.

Throughout this section we use the following notation.

Definition 5.17. For every $s \in 2^{<\omega}$, set $N_{s}=\left\{x \in 2^{\omega}: s \sqsubseteq x\right\}$. Let $\Omega=2^{<\omega}$. We define $\Phi: \mathcal{K}\left(2^{\omega}\right) \rightarrow \mathcal{P}(\Omega)$,

$$
\Phi(K)=\left\{s \in \Omega: N_{s} \cap K \neq \emptyset\right\} .
$$


For every $\mathcal{A} \subseteq \mathcal{P}(\Omega)$, set $\mathcal{A}^{\downarrow}=\{B \in \mathcal{P}(\Omega): \exists A \in \mathcal{A}(B \subseteq A)\}$. For every $\mathcal{I} \subseteq \mathcal{K}\left(2^{\omega}\right)$ we define $\mathfrak{I}=(\Phi[\mathcal{I}])^{\downarrow}$.

Lemma 5.18. With the notation of Definition 5.17, we have the following:

(1) $\Phi$ is continuous, and for every $K, L \in \mathcal{K}\left(2^{\omega}\right), K \subseteq L \Leftrightarrow \Phi(K) \subseteq \Phi(L)$. In particular, $\Phi: \mathcal{K}\left(2^{\omega}\right) \rightarrow \Phi\left[\mathcal{K}\left(2^{\omega}\right)\right]$ is a homeomorphism.

(2) If $\mathcal{I} \subseteq \mathcal{K}\left(2^{\omega}\right)$ is an ideal, then $\mathfrak{I} \subseteq \mathcal{P}(\Omega)$ is also an ideal.

(3) If $\mathcal{A} \subseteq \mathcal{P}(\Omega)$ is analytic, then $\mathcal{A}^{\downarrow} \subseteq \mathcal{P}(\Omega)$ is also analytic.

(4) If $\mathcal{I} \subseteq \mathcal{K}\left(2^{\omega}\right)$ is an analytic ideal, then $\mathfrak{I} \subseteq \mathcal{P}(\Omega)$ is also an analytic ideal.

(5) 3 and 4 also hold if we replace "analytic" with " $F_{\sigma}$ ".

Proof. It is obvious that for every $K, L \in \mathcal{K}\left(2^{\omega}\right), K \subseteq L \Leftrightarrow \Phi(K) \subseteq \Phi(L)$. In particular, $\Phi$ is injective. For every $s \in \Omega, \Phi^{-1}(\{A \in \mathcal{P}(\Omega): s \in A\})=\{K \in$ $\left.\mathcal{K}\left(2^{\omega}\right): K \cap N_{s} \neq \emptyset\right\}$ is clopen in the Vietoris topology, thus $\Phi$ is continuous. Since $\mathcal{K}\left(2^{\omega}\right)$ is compact, $\Phi$ is a homeomorphism.

For 2] it is obvious that $\mathfrak{I}$ is closed downward. To see that it is closed under taking unions, pick $A_{i} \in \mathfrak{I}(i<2)$. Then there are $K_{i} \in \mathcal{I}(i<2)$ such that $A_{i} \subseteq \Phi\left(K_{i}\right)(i<2)$. By 1, $A_{0} \cup A_{1} \subseteq \Phi\left(K_{0}\right) \cup \Phi\left(K_{1}\right)=\Phi\left(K_{0} \cup K_{1}\right)$, so the statement follows.

To see 3 observe that $\{(A, B) \in \mathcal{P}(\Omega) \times \mathcal{P}(\Omega): B \subseteq A\}$ is a closed relation and $\mathcal{A}^{\downarrow}$ is the projection of $\{(A, B) \in \mathcal{P}(\Omega) \times \mathcal{P}(\Omega): B \subseteq A \in \mathcal{A}\}$ to the second coordinate. Hence $\mathcal{A}^{\downarrow}$ is analytic if $\mathcal{A}$ is analytic, and by $\mathcal{P}(\Omega)$ being compact, $\mathcal{A}^{\downarrow}$ is $F_{\sigma}$ if $\mathcal{A}$ is $F_{\sigma}$.

Finally, 4 and 5 follow from 1, 2 and 3 .

The ideals $\mathcal{I}$ and $\mathfrak{I}$ are cofinally similar, as well.

Lemma 5.19. Let $\mathcal{I} \subseteq \mathcal{K}\left(2^{\omega}\right)$ be an analytic ideal. Then $\mathcal{I} \equiv_{T} \mathfrak{I}$. Moreover:

(1) $\Phi: \mathcal{I} \rightarrow \mathfrak{I}$ and $\Phi^{-1}: \Phi[\mathcal{I}] \rightarrow \mathcal{I}$ are continuous Tukey maps;

(2) there is a Tukey reduction $\Phi^{-1} \circ f: \mathfrak{I} \rightarrow \mathcal{I}$ where $f: \mathfrak{I} \rightarrow \mathfrak{I}$ is a Souslin measurable Tukey map such that $H \subseteq f(H) \in \Phi[\mathcal{I}](H \in \mathfrak{I})$.

Proof. To see 1 , let $\mathcal{H} \subseteq \mathcal{I}$ be an arbitrary set. If $\Phi[\mathcal{H}] \subseteq \mathfrak{I}$ is bounded, then there is an $L \in \mathcal{I}$ such that $\Phi(K) \subseteq \Phi(L)(K \in \mathcal{H})$. By Lemma 5.1811 this implies $K \subseteq L$ $(K \in \mathcal{H})$, i.e. $\mathcal{H}$ is also bounded. Hence $\Phi$ is a Tukey map, which is continuous by Lemma 5.1811. The argument for $\Phi^{-1}$ is identical.

For 2], consider the set $P=\{(A, B) \in \mathfrak{I} \times \Phi[\mathcal{I}]: A \subseteq B\}$. Then $P \subseteq \mathcal{P}(\Omega) \times \mathcal{P}(\Omega)$ is analytic, so by the Jankov-von Neumann Uniformization Theorem (see e.g. [11, Theorem 18.1, p. 120]) $P$ has a Souslin measurable uniformizing function $f: \mathfrak{I} \rightarrow$ $\Phi[\mathcal{I}]$. Also, $H \subseteq f(H) \in \Phi[\mathcal{I}](H \in \mathfrak{I})$ holds.

Since $f$ pointwise dominates the identity function, it is a Tukey map. Then by 1. $\Phi^{-1} \circ f: \mathfrak{I} \rightarrow \mathcal{I}$ is also a Tukey map, which completes the proof.

Corollary 5.20. Let $\mathcal{I} \subseteq \mathcal{K}\left(2^{\omega}\right)$ be an analytic ideal.

(1) For every cardinal $\kappa$ we have $[\kappa]^{<\omega} \leq_{T} \mathcal{I} \Leftrightarrow[\kappa]^{<\omega} \leq_{T} \mathfrak{I}$.

(2) There exists a non-empty perfect strongly unbounded subset of $\mathcal{I}$ if and only if there exists a non-empty perfect strongly unbounded subset of $\mathfrak{I}$.

Proof. Statement 1 follows from Lemma 5.19] To see 2 suppose first that $P \subseteq \mathcal{I}$ is a non-empty perfect strongly unbounded set. By Lemma 5.1811, $\Phi$ is continuous 
and injective. So $\Phi[P]$ is also non-empty and perfect. By Lemma [5.19]1, $\Phi$ is a Tukey map, i.e. by Lemma 5.10, $\Phi[P]$ is strongly unbounded.

Finally let $P \subseteq \mathfrak{I}$ be a non-empty perfect strongly unbounded set. Let $f$ be the function of Lemma 5.19|2, Then $\left.f\right|_{P}$ is Souslin measurable. Hence it has the Baire property, so by passing to a non-empty perfect subset of $P$ we can assume that $\left.f\right|_{P}$ is continuous (see e.g. [11, Theorem 8.38, p. 52]).

By Lemma $\left[5.10\right.$ and Lemma [5.19/2, $\left.f\right|_{P}$ is finite-to one. Thus $f[P] \subseteq \Phi[\mathcal{I}]$ is an uncountable compact strongly unbounded set; in particular, it has a non-empty perfect subset $Q$, which is also strongly unbounded. So by Lemma 5.1911 and Lemma 5.10, $\Phi^{-1}(Q) \subseteq \mathcal{I}$ is a non-empty perfect strongly unbounded set.

Our main theorem is the following.

Theorem 5.21. There is an analytic ideal $\mathcal{J} \subseteq \mathcal{K}\left(2^{\omega}\right)$ with the following properties:

(1) $\left[\omega_{1}\right]^{<\omega} \leq_{T} \mathcal{J}$.

(2) $\left[\omega_{2}\right]^{<\omega} \mathbb{Z}_{T} \mathcal{J}$.

(3) There is no non-empty perfect strongly unbounded subset of $\mathcal{J}$.

The following corollary answers [16, Conjecture 2, p. 194] in the negative.

Corollary 5.22. There is an analytic ideal $\mathfrak{J} \subseteq \mathcal{P}(\omega)$ with the following properties:

(1) $\left[\omega_{1}\right]^{<\omega} \leq_{T} \mathfrak{J}$.

(2) $\left[\omega_{2}\right]^{<\omega} \mathbb{Z}_{T} \mathfrak{J}$.

(3) There is no non-empty perfect strongly unbounded subset of $\mathfrak{J}$.

Proof. Let $\mathcal{J}$ be the ideal of Theorem 5.21 and set $\mathfrak{J}=(\Phi[\mathcal{J}])^{\downarrow}$. By Lemma 5.184, $\mathfrak{J}$ is an analytic ideal. By Corollary 5.20, 1,2 and 3 follows from Theorem 5.21,

Also, Theorem 5.7/2 easily follows.

Proof of Theorem 5.722, Let $\mathfrak{J}$ be the ideal of Corollary 5.22, Assume the Continuum Hypothesis holds. Then $\mathcal{I}_{\max } \equiv_{T}\left[\omega_{1}\right]^{<\omega} \leq_{T} \mathfrak{J}$ by Corollary [5.2211,

Let $f: \mathcal{I}_{\max } \rightarrow \mathfrak{J}$ be an arbitrary Tukey map. By Lemma [5.10, $f\left[\mathcal{I}_{\max }\right] \subseteq \mathfrak{J}$ is strongly unbounded, so every subset of $f\left[\mathcal{I}_{\max }\right]$ is strongly unbounded, as well. Hence by Corollary [5.2213, $f\left[\mathcal{I}_{\max }\right]$ has no non-empty perfect subsets.

If $f$ is either Lebesgue measurable or has the Baire property, then there is an uncountable Borel set $B \subseteq \mathcal{I}_{\max }$ such that $\left.f\right|_{B}$ is continuous. By Lemma 5.10, $f[B] \subseteq \mathfrak{J}$ is an uncountable strongly unbounded analytic set; in particular, it has a non-empty perfect strongly unbounded subset. This contradicts Corollary 5.2213,

It remains to prove Theorem 5.21, Recall the definition of the Cantor-Bendixson derivative (see e.g. [11, Definition 6.10, p. 33]): for every $K \in \mathcal{K}\left(2^{\omega}\right)$, set $K^{\prime}=\{x \in$ $\left.K: x \in \operatorname{cl}_{2} \omega(K \backslash\{x\})\right\}$. We define, by induction on $n<\omega$, the iterated derivatives: $K^{(0)}=K, K^{(n+1)}=\left(K^{(n)}\right)^{\prime}(n<\omega)$. Also recall that there exists an analytic equivalence relation $E \subseteq 2^{\omega} \times 2^{\omega}$ such that $E$ has exactly $\omega_{1}$ many equivalence classes (see e.g. [9, Exercise 32.3, p. 625]).

Definition 5.23. Set $\mathcal{R}=\left\{K \in \mathcal{K}\left(2^{\omega}\right): \exists n<\omega\left(K^{(n)}=\emptyset\right)\right\}$. Let $E \subseteq 2^{\omega} \times 2^{\omega}$ be an analytic equivalence relation with exactly $\omega_{1}$ many equivalence classes. We 
define

$$
\mathcal{L}=\left\{K \in \mathcal{K}\left(2^{\omega}\right): K \in \mathcal{R}, \forall x, y \in K((x, y) \in E)\right\} .
$$

It is known that $\mathcal{R}$ is a Borel subset of $\mathcal{K}\left(2^{\omega}\right)$ (see [3] and [4 for its exact Borel class). However, due to the universal quantification in the definition of $\mathcal{L}$, the following is not straightforward.

Proposition 5.24. $\mathcal{L} \subseteq \mathcal{K}\left(2^{\omega}\right)$ is analytic.

Proof. For every $n<\omega$, let $\vartheta_{n}: \mathcal{K}\left(2^{\omega}\right)^{n} \rightarrow \mathcal{K}\left(2^{\omega}\right)$ denote the union function, i.e. for every $\left(K_{i}\right)_{i<n} \in \mathcal{K}\left(2^{\omega}\right)^{n}, \vartheta_{n}\left(\left(K_{i}\right)_{i<n}\right)=\bigcup_{i<n} K_{i}$. It is clear that $\vartheta_{n}(n<\omega)$ are continuous.

Recall $N_{s}=\left\{\sigma \in 2^{\omega}: s \sqsubseteq \sigma\right\}\left(s \in 2^{<\omega}\right)$. We define $\mathcal{L}_{n}, \mathcal{L}_{n}^{+} \subseteq \mathcal{K}\left(2^{\omega}\right)$ by induction on $n$, as follows. Set $\mathcal{L}_{0}=\left[2^{\omega}\right] \leq 1$. If $n<\omega$ and $\mathcal{L}_{n}$ is already defined, set

$$
\mathcal{L}_{n}^{+}=\{\emptyset\} \cup \bigcup_{m<\omega} \vartheta_{m}\left[\left\{\left(L_{i}\right)_{i<m}: \forall i<m \exists u_{i} \in L_{i}\left(\left(u_{i}, u_{j}\right) \in E(i, j<m)\right)\right\}\right]
$$

and

$\mathcal{L}_{n+1}=\mathcal{L}_{0} \cup\left\{L \in \mathcal{K}\left(2^{\omega}\right): \exists u, v \in L\left(u \neq v,(u, v) \in E, L \backslash N_{\left.u\right|_{m}} \in \mathcal{L}_{n}^{+}(m<\omega)\right)\right\}$.

It follows from the definition that $\mathcal{L}_{n}, \mathcal{L}_{n}^{+} \subseteq \mathcal{K}\left(2^{\omega}\right)(n<\omega)$ are analytic sets and $\mathcal{L}_{n} \subseteq \mathcal{L}_{n}^{+}(n<\omega)$. So the proof will be complete if we show $\mathcal{L}=\bigcup_{n<\omega} \mathcal{L}_{n}^{+}$.

To see $\bigcup_{n<\omega} \mathcal{L}_{n}^{+} \subseteq \mathcal{L}$, we show by induction on $n$ that for every $L \in \mathcal{L}_{n}^{+}$we have $L^{(n+1)}=\emptyset$ and $(x, y) \in E(x, y \in L)$. The statement is obvious for $n=0$; so let $n<\omega$ be arbitrary and suppose that the statement holds for $\mathcal{L}_{n}^{+}$. Let $L \in \mathcal{L}_{n+1}^{+}$be arbitrary. Suppose first $L \in \mathcal{L}_{n+1}$, and let $u, v \in L$ be as in (5.3). By the inductive hypothesis, $L^{(n)} \subseteq\{u\}$ and $(x, y) \in E(x, y \in L \backslash\{u\})$. Thus $L^{(n+1)}=\emptyset$, and $(u, v) \in E, u \neq v$ imply $(x, y) \in E(x, y \in L)$.

In the general case, for some $m<\omega$, let $L=\bigcup_{i<m} L_{i}$ where $L_{i} \in \mathcal{L}_{n+1}(i<m)$. Since $L_{i}^{(n+1)}=\emptyset(i<m)$, we have $L^{(n+1)}=\emptyset$. Let $u_{i} \in L_{i}(i<m)$ be as in (5.2). Since $(x, y) \in E\left(x, y \in L_{i}, i<m\right)$ and $\left(u_{i}, u_{j}\right) \in E(i, j<m)$, we get $(x, y) \in E$ $(x, y \in L)$, as required.

To see $\mathcal{L} \subseteq \bigcup_{n<\omega} \mathcal{L}_{n}^{+}$, we show by induction on $n$ that for every $L \in \mathcal{L}, L^{(n+1)}=\emptyset$ implies $L \in \mathcal{L}_{n}^{+}$. For $n=0, L^{\prime}=\emptyset$ holds if and only if $L$ is finite; then $L \in \mathcal{L}_{0}^{+}$. So let $n<\omega$ and suppose that the statement holds for $n$. Let $L \in \mathcal{L}$ be arbitrary satisfying $L^{(n+2)}=\emptyset$. If $L \in \mathcal{L}_{0}$, then $L \in \mathcal{L}_{n+1}$ follows. If $L \notin \mathcal{L}_{0}$ and $L^{(n+1)}=\emptyset$, then let $u, v \in L$ be arbitrary with $u \neq v$. By $L \in \mathcal{L}$, we have $(u, v) \in E$. By $L^{(n+1)}=\emptyset$ and by the inductive assumption, $L \backslash N_{\left.u\right|_{m}} \in \mathcal{L}_{n}^{+}(m<\omega)$, so $L \in \mathcal{L}_{n+1}$.

If $L^{(n+1)}$ is a singleton, then set $\{u\}=L^{(n+1)}$ and let $v \in L \backslash\{u\}$ be arbitrary. As in the previous case, by the inductive assumption, $L \backslash N_{\left.u\right|_{m}} \in \mathcal{L}_{n}^{+}(m<\omega)$, so $L \in \mathcal{L}_{n+1}$.

Finally, if $\left|L^{(n+1)}\right|>1$, then it is a finite set, say $L^{(n+1)}=\left\{u_{i}: i<m\right\}$ for some $m<\omega$. Let $L_{i} \in \mathcal{K}\left(2^{\omega}\right)(i<m)$ be arbitrary satisfying $u_{i} \in L_{i} \subseteq L \backslash\left\{u_{j}: j<\right.$ $m, j \neq i\}(i<m)$ and $L=\bigcup_{i<m} L_{i}$. Since $L_{i}^{(n+1)}=\left\{u_{i}\right\}(i<m)$, we have $L_{i} \in \mathcal{L}_{n+1}(i<m)$. By $L \in \mathcal{L},\left(u_{i}, u_{j}\right) \in E(i, j<m)$; hence $L \in \mathcal{L}_{n+1}^{+}$, as stated. 
Proof of Theorem [5.21. Recall that for every $n<\omega, \vartheta_{n}: \mathcal{K}\left(2^{\omega}\right)^{n} \rightarrow \mathcal{K}\left(2^{\omega}\right)$ denotes the union function. With the set $\mathcal{L}$ defined in (5.1), set

$$
\mathcal{J}=\bigcup_{n<\omega} \vartheta_{n}\left[\mathcal{L}^{n}\right]
$$

We show that $\mathcal{J}$ fulfills the requirements.

Since $\mathcal{L}$ is closed downward and $\mathcal{J}$ is closed under taking finite unions, $\mathcal{J}$ is an ideal of compact sets. The functions $\vartheta_{n}(n<\omega)$ are continuous. Hence by Proposition 5.24 $\mathcal{J}$ is analytic. It is immediate from the definition that for every $K \in \mathcal{K}\left(2^{\omega}\right), K \in \mathcal{J}$ if and only if

(i) $K$ intersects only finitely many equivalence classes of $E$;

(ii) for every equivalence class $C \subseteq 2^{\omega}$ of $E, K \cap C \in \mathcal{K}\left(2^{\omega}\right)$ and $(K \cap C)^{(n)}=\emptyset$ for some $n<\omega$.

Let $H \in\left[2^{\omega}\right]^{\omega_{1}}$ be an arbitrary set such that $(x, y) \notin E(x, y \in H)$, and set $\mathcal{H}=[H]^{1}$. By (i) , $\mathcal{H} \subseteq \mathcal{J}$ is strongly unbounded, i.e., by Proposition [5.9, 1 holds.

To see 2, by Proposition 5.9 we have to show that if $\mathcal{H} \in[\mathcal{J}]^{\omega_{2}}$, then $\mathcal{H}$ is not strongly unbounded. So let $\mathcal{H} \in[\mathcal{J}]^{\omega_{2}}$ be arbitrary. Let $\left\{E_{\alpha}: \alpha<\omega_{1}\right\}$ be an enumeration of the equivalence classes of $E$. By (ii) and (iii), there are $n<\omega$, $A \in\left[\omega_{1}\right]^{<\omega}$ and $\mathcal{H}^{\prime} \in[\mathcal{H}]^{\omega_{2}}$ such that for every $K \in \mathcal{H}^{\prime}$,

$$
K \cap E_{\alpha} \neq \emptyset \Leftrightarrow \alpha \in A \text { and }\left(K \cap E_{\alpha}\right)^{(n)}=\emptyset(\alpha \in A) .
$$

Consider the space $\mathcal{X}=\prod_{\alpha \in A}\left(\mathcal{J} \cap \mathcal{K}\left(E_{\alpha}\right)\right)$ and the set

$$
X=\left\{\left(K_{\alpha}\right)_{\alpha \in A}: K_{\alpha} \in \mathcal{J} \cap \mathcal{K}\left(E_{\alpha}\right), \bigcup_{\alpha \in A} K_{\alpha} \in \mathcal{H}^{\prime}\right\} .
$$

Then $\mathcal{X}$ is a separable metric space and $X \subseteq \mathcal{X}$ is an uncountable set. Since $\mathcal{X}$ is Lindelöf, there is a $\left(K_{\alpha}\right)_{\alpha \in A} \in \mathcal{X}$ and an injective sequence $\left(K_{\alpha}(m)\right)_{\alpha \in A} \in X$ $(m<\omega)$ such that $\lim _{m<\omega} K_{\alpha}(m)=K_{\alpha}(\alpha \in A)$. Set $H(m)=\bigcup_{\alpha \in A} K_{\alpha}(m)$ $(m<\omega), H=\bigcup_{\alpha \in A} K_{\alpha}$ and $L=H \cup \bigcup_{n<\omega} H(m)$. Observe that $H \in \mathcal{J}$; in particular, there is a $d<\omega$ such that $H^{(d)}=\emptyset$. Also, $H(m) \in \mathcal{H}^{\prime}$ implies $H(m)^{(n)}=\emptyset(m<\omega)$.

We have $L \cap E_{\alpha} \neq \emptyset \Leftrightarrow \alpha \in A\left(\alpha<\omega_{1}\right)$ and $L \cap E_{\alpha} \in \mathcal{K}\left(2^{\omega}\right)(\alpha \in A)$. Moreover, $L^{(n)} \subseteq H$, hence $L^{(n+d)}=\emptyset$. By (ii) and (iii) this implies $L \in \mathcal{J}$. Since $\left(\left(K_{\alpha}(m)\right)_{\alpha \in A}\right)_{m<\omega}$ is injective, $\{H(m): m<\omega\} \subseteq \mathcal{H}$ is an infinite set. By $H(m) \subseteq L(m<\omega), \mathcal{H}$ is not strongly unbounded, as stated.

Finally, observe that by Shoenfield's Absoluteness (see e.g. [9, Theorem 25.20, p. 490]), a non-empty perfect strongly unbounded subset of $\mathcal{J}$ would remain strongly unbounded in any forcing extension. Hence 3 follows from 2

5.4. Miscellanea. In the previous sections we restricted our investigations to analytic ideals $\mathcal{I} \subseteq(\mathcal{P}(\omega), \subseteq)$. Unfortunately, there are simple directed partial orders which are not cofinally similar to any such ideal. The following may be considered as a negative answer to [16, Question 1, p. 193].

Proposition 5.25. Let 1 denote the one element set with the trivial order, let $\omega$ denote the first infinite ordinal with its usual well-order, and let $\left(\omega^{\omega}, \leq^{\star}\right)$ be the set 
of all functions from $\omega$ to $\omega$, partially ordered by eventual dominance.

(1) If $\mathcal{I} \subseteq(\mathcal{P}(\omega), \subseteq)$ is an arbitrary directed set, then $\mathcal{I} \leq_{T} 1$ or $\omega \leq_{T} \mathcal{I}$.

(2) The directed partial order $\left(\omega^{\omega}, \leq^{\star}\right)$ satisfies $\leq^{\star} \subseteq \omega^{\omega} \times \omega^{\omega}$ is $F_{\sigma}$, but it is incomparable with $\omega$ in the Tukey order. In particular, $\left(\omega^{\omega}, \leq^{\star}\right) \not \equiv{ }_{T} \mathcal{I}$ for every directed set $\mathcal{I} \subseteq(\mathcal{P}(\omega), \subseteq)$.

Proof. To see 1, let $S=\bigcup I$. If $S \in I$, then $I \leq_{T} 1$. If $S \notin I$, let $\left(S_{n}\right)_{n<\omega} \subseteq I$ be an increasing sequence satisfying $S=\bigcup_{n<\omega} S_{n}$; such a sequence exists since $I$ is directed. Then $f: \omega \rightarrow I, f(n)=S_{n}(n<\omega)$ is a Tukey map.

For 2, $\omega \mathbb{L}_{T}\left(\omega^{\omega}, \leq^{\star}\right)$ follows from the fact that every countable subset of $\left(\omega^{\omega}, \leq^{\star}\right)$ is bounded. To see $\left(\omega^{\omega}, \leq^{\star}\right) \leq_{T} \omega$ let $f:\left(\omega^{\omega}, \leq^{\star}\right) \rightarrow \omega$ be an arbitrary function. Then there is an $n<\omega$ such that $f^{-1}(\{n\}) \subseteq \omega^{\omega}$ is non-meager. Since for every $\varphi \in \omega^{\omega},\left\{\psi \in \omega^{\omega}: \psi \leq^{\star} \varphi\right\}$ is meager, such a set cannot be bounded. Hence $f$ is not a Tukey map. The second statement follows from 1 .

On the other hand, closed directed partial orders on analytic spaces admit representations as analytic ideals on $\omega$.

Proposition 5.26. Let $(I, \leq)$ be a topological space endowed with a directed partial order such that $I$ is a continuous image of a Polish space and $\leq \subseteq I \times I$ is closed. Then there is an analytic ideal $\mathfrak{I} \subseteq(\mathcal{P}(\omega), \subseteq)$ such that $(I, \leq) \equiv_{T} \mathfrak{I}$.

Proof. Let $G \subseteq 2^{\omega}$ be an arbitrary set homeomorphic to $\omega^{\omega}$, and let $f: G \rightarrow I$ be a continuous surjection (see e.g. [11, Theorem 7.9, p. 38]). For every $a \in I$, set $L_{a}=\{b \in I: b \leq a\}$. Note that $L_{a} \subseteq I(a \in I)$ are closed. We show that

$$
\mathcal{I}=\left\{K \in \mathcal{K}\left(2^{\omega}\right): \exists a \in I\left(K \subseteq \operatorname{cl}_{2} \omega\left(f^{-1}\left(L_{a}\right)\right)\right\}\right.
$$

is an analytic ideal of compact subsets of $2^{\omega}$ such that $(I, \leq) \equiv_{T} \mathcal{I}$. Then, with the notation of Definition 5.17 , by Lemma $5.19 \mathfrak{I}=(\Phi[\mathcal{I}])^{\downarrow}$ fulfills the requirements.

It is obvious that $\mathcal{I}$ is closed downward. Since $I$ is directed, $\mathcal{I}$ is closed under taking finite unions. Next we show that $\mathcal{I}$ is analytic. Let $\mathcal{F}(G)$ denote the set of closed subsets of $G$ endowed with the Effros Borel structure (see e.g. 11, Section 12.C, p. 75]). Recall that a sub-basis of the corresponding topology consists of a set of the form $\{F \in \mathcal{F}(G): F \cap U \neq \emptyset\}$, where $U$ varies over the open subsets of $G$. First we show that

$$
R=\left\{(F, a) \in \mathcal{F}(G) \times I: F \subseteq f^{-1}\left(L_{a}\right)\right\}
$$

is a closed set. Let $(F, a) \in \mathcal{F}(G) \times I,\left(F_{n}, a_{n}\right) \in R(n<\omega)$ be such that $\lim _{n<\omega}\left(F_{n}, a_{n}\right)=(F, a)$. Let $x \in F$ be arbitrary; then by $F_{n} \subseteq f^{-1}\left(L_{a_{n}}\right)(n<\omega)$ there are $x_{n} \in f^{-1}\left(L_{a_{n}}\right)(n<\omega)$ such that $\lim _{n<\omega} x_{n}=x$. Since $f$ is continuous, $f(x)=\lim _{n<\omega} f\left(x_{n}\right)$. By $\leq \subseteq I \times I$ being closed, $f\left(x_{n}\right) \leq a_{n}(n<\omega)$ implies $f(x) \leq a$. Thus $x \in f^{-1}\left(L_{a}\right)$. Since $x \in F$ was arbitrary, $F \subseteq f^{-1}\left(L_{a}\right)$; thus $(F, a) \in R$ follows.

Observe that $\left\{(K, F) \in \mathcal{K}\left(2^{\omega}\right) \times \mathcal{F}(G): K \subseteq \operatorname{cl}_{2^{\omega}}(F)\right\}$ is a Borel set (see e.g. the proof of [11, Theorem 12.6, p. 75]). Hence

$$
S=\left\{(K, F, a) \in \mathcal{K}\left(2^{\omega}\right) \times \mathcal{F}(G) \times I: K \subseteq \operatorname{cl}_{2} \omega(F), F \subseteq f^{-1}\left(L_{a}\right)\right\}
$$

is also Borel. It is obvious that

$$
T=\left\{(K, a) \in \mathcal{K}\left(2^{\omega}\right) \times I: K \subseteq \operatorname{cl}_{2^{\omega}}\left(f^{-1}\left(L_{a}\right)\right)\right\}=\operatorname{Pr}_{\mathcal{K}\left(2^{\omega}\right) \times I}(S),
$$

so $T$ is an analytic set. Since $I$ is analytic, $\mathcal{I}=\operatorname{Pr}_{\mathcal{K}\left(2^{\omega}\right)}(T)$ is analytic, as well. 
We show that $g: I \rightarrow \mathcal{I}, g(a)=\operatorname{cl}_{2} \omega\left(f^{-1}\left(L_{a}\right)\right)(a \in I)$ is a Tukey map. Let $A \subseteq I$ be arbitrary. If $g[A] \subseteq \mathcal{I}$ is bounded, then there is a $b \in I$ satisfying $\operatorname{cl}_{2^{\omega}}\left(f^{-1}\left(L_{a}\right)\right) \subseteq \operatorname{cl}_{2^{\omega}}\left(f^{-1}\left(L_{b}\right)\right)(a \in A)$. Then $f^{-1}(a) \subseteq \operatorname{cl}_{2^{\omega}}\left(f^{-1}\left(L_{b}\right)\right) \cap G=$ $f^{-1}\left(L_{b}\right)(a \in A)$, so $a \leq b(a \in A)$. Thus $g$ maps unbounded sets into unbounded sets, as stated.

Finally, let $h: \mathcal{I} \rightarrow I$ be any function satisfying $K \subseteq \operatorname{cl}_{2} \omega\left(f^{-1}\left(L_{h(K)}\right)\right)(K \in \mathcal{I})$; we show that $h$ is a Tukey map. Let $\mathcal{A} \subseteq \mathcal{I}$ be arbitrary. If $h[\mathcal{A}] \subseteq I$ is bounded, then there is a $b \in I$ satisfying $h(K) \leq b$; hence $L_{h(K)} \subseteq L_{b}(K \in \mathcal{A})$. Then $K \subseteq \operatorname{cl}_{2} \omega\left(f^{-1}\left(L_{b}\right)\right)(K \in \mathcal{A})$; i.e. $h$ maps unbounded sets into unbounded sets, as required.

Next we present a simple proof of Proposition 5.5.

Proof of Proposition 5.5. Observe that for every $n<\omega,\left[2^{\omega}\right] \leq n \subseteq \mathcal{K}\left(2^{\omega}\right)$ is closed. Hence $\left[2^{\omega}\right]^{<\omega} \subseteq \mathcal{K}\left(2^{\omega}\right)$ is an $F_{\sigma}$ ideal of compact sets. Then, with the notation of Definition 5.17 by Lemma 5.1815] and Lemma 5.19, $\mathcal{I}_{\max }=\left(\Phi\left[\left[2^{\omega}\right]^{<\omega}\right]\right)^{\downarrow}$ fulfills the requirements.

As we mentioned above, $\mathcal{I}_{\max }$ is not basic. To see this, let us recall the definition of basic directed partial orders. Recall that a set is called bounded if it is bounded from above.

Definition 5.27 (25, Definition, p. 1881]). Let $(D, \leq)$ be a separable metric space endowed with a partial order. Then $(D, \leq)$ is basic if

(1) each pair of elements of $D$ has the least upper bound with respect to $\leq$, and the binary operation of least upper bound from $D \times D$ to $D$ is continuous;

(2) each bounded sequence has a converging subsequence;

(3) each converging sequence has a bounded subsequence.

Since every infinite subset of $\mathcal{I}_{\max }$ is unbounded, in a topology making $\mathcal{I}_{\max }$ satisfy Definition $5.27 / 3$ there are no injective convergent sequences. The only such metric topology is the discrete topology, which is not separable in this case.

We close this section with the proof of Proposition [5.4. The construction originates from [16. Theorem 6, p. 183], and an analogous construction was used in [17] to show that the structure $\left(\mathcal{P}(\omega), \subseteq^{\star}\right)$ embeds into the family of $F_{\sigma}$ ideals on $\omega$ partially ordered by Borel reduction. Our main improvement compared to [16] and [17, which makes it possible to omit definability assumptions, is that our proof for non-reducibility is purely combinatorial.

We define sequences $\left(b_{j}\right)_{j<\omega}$ and $\left(m_{j}\right)_{j<\omega}$ by induction on $j$, as follows. Set $m_{0}=0$; if $j<\omega$ and $m_{j}$ is already defined, set $b_{j}=2^{j \cdot m_{j}}$ and $m_{j+1}=m_{j}+b_{j}$.

Let $I_{j}=\left[m_{j}, m_{j+1}\right)(j<\omega)$. Let log stand for a logarithm of base 2. For every $S \in[\omega]^{\omega}$ and $N<\omega$, let $I_{S}(N)=\bigcup_{j \in S \cap N} I_{j}$ and $I_{S}=\bigcup_{j \in S} I_{j}$. For every $j<\omega$ and $x \subseteq \omega$,

$$
\|x\|_{j}=\frac{\log \left(\left|x \cap I_{j}\right|+1\right)}{m_{j}+1},\|x\|=\sup _{j<\omega}\|x\|_{j} .
$$

We define $\mathcal{F}_{S}=\left\{x \subseteq I_{S}: \sup _{j<\omega}\|x\|_{j}<\infty\right\}$. For every $N<\omega$, let $\mathcal{F}_{S}(N)=$ $\left\{x \in \mathcal{F}_{S}:\|x\| \leq N\right\}$. We will use the property that for arbitrary $n, j<\omega$ and $\left(x_{i}\right)_{i<n} \subseteq \mathcal{P}(\omega)$,

$$
\left\|\bigcup_{i<n} x_{i}\right\|_{j} \leq \sup _{i<n}\left\|x_{i}\right\|_{j}+\frac{\log (n)}{m_{j}+1}
$$


Proposition 5.4 is an immediate corollary of the following two statements.

Proposition 5.28. For every $S \in[\omega]^{\omega}, \mathcal{F}_{S} \subseteq \mathcal{P}(\omega)$ is an $F_{\sigma}$ ideal which is not basic in any topology.

Proposition 5.29. For every $S, T \in[\omega]^{\omega}, \mathcal{F}_{S} \leq_{T} \mathcal{F}_{T}$ if and only if $S \subseteq^{\star} T$.

Proof of Proposition 5.28. It is obvious that $\mathcal{F}_{S}(N) \subseteq \mathcal{P}(\omega)(N<\omega)$ are closed sets, so $\mathcal{F}_{S}$ is an $F_{\sigma}$ ideal. Suppose there is a topology on $\mathcal{F}_{S}$ which makes it basic. For every $N<\omega$, let $\left(x_{i}(N)\right)_{i<\omega} \subseteq[\omega]^{<\omega}$ be a sequence such that

(1) for every $i<\omega$ we have $x_{i}(N) \subseteq I_{S}, N-1 \leq\left\|x_{i}(N)\right\| \leq N$;

(2) for every $j<\omega$ we have $\left|\left\{i<\omega: x_{i}(N) \cap I_{j} \neq \emptyset\right\}\right| \leq 1$.

Set $X_{i}(N)=\bigcup_{i \leq k<\omega} x_{k}(N)(i, N<\omega)$. Then by 1 and 2 we have $X_{i}(N) \subseteq I_{S}$ and $\left\|X_{i}(N)\right\| \leq N$, i.e. $X_{i}(N) \in \mathcal{F}_{S}(i, N<\omega)$.

Fix $N<\omega$. The sequence $\left(x_{k}(N)\right)_{k<\omega}$ is bounded by $X_{0}(N)$, so by Definition 5.272 it has a convergent subsequence $\left(x_{k}(N)\right)_{k \in I_{N}}$ for some $I_{N} \in[\omega]^{\omega}$.

By [25, Lemma 3.1, p. 1882], for every $i<\omega, X_{i}^{+}(N)=\left\{y \in \mathcal{F}_{S}: y \subseteq X_{i}(N)\right\}$ is compact. Since the convergent sequence $\left(x_{k}(N)\right)_{k \in I_{N}}$ is eventually a subsequence of $X_{i}^{+}(N)(i<\omega), \lim _{k \in I_{N}} x_{k}(N) \in \bigcap_{i<\omega} X_{i}^{+}(N)=\{\emptyset\}$. Thus $\lim _{i \in I_{N}} x_{i}(N)=\emptyset$ $(N<\omega)$.

Since a basic topology is metric, by an easy diagonalization argument we can find a function $\varphi: \omega \rightarrow \omega$ such that $\varphi(N) \in I_{N}(N<\omega)$ and $\lim _{N<\omega} x_{\varphi(N)}(N)=$ $\emptyset$. Thus $\left(x_{\varphi(N)}(N)\right)_{N<\omega}$ is a convergent sequence, which by (1) has no bounded subsequence. This contradicts Definition $5.27 / 3$.

The non-reduction part of Proposition 5.29 is based on the following. For every $t \in 2^{\leq \omega}$ we set $[t=1]=\{n<\omega: t(n)=1\}$. Recall $N_{s}=\{x \in \mathcal{P}(\omega): x \cap|s|=[s=$ 1]\} $\left(s \in 2^{<\omega}\right)$.

Lemma 5.30. Let $S, T \in[\omega]^{\omega}$ satisfy $S \cap T=\emptyset$. Let $A \subseteq \mathcal{F}_{S}(1), n \in S$ and $s \in 2^{m_{n}}$ be such that $N_{s} \cap \mathcal{F}_{S}(1) \neq \emptyset$ and let $A$ be of second category everywhere in $N_{s} \cap \mathcal{F}_{S}(1)$ in the relative topology of $\mathcal{F}_{S}(1)$. Let $N<\omega$, and let $f: A \rightarrow \mathcal{F}_{T}(N)$ be an arbitrary function. Then there is a $t \in 2^{m_{n}}$ such that $[t=1] \subseteq T,\|t\| \leq N$, $B=\left\{x \in A:\left.f(x)\right|_{m_{n}}=t\right\} \subseteq \mathcal{F}_{S}(1)$ is of second category and $\|\cup B\|_{n} \geq n-2$.

Proof. Let $\mathcal{T}=\left\{t \in 2^{m_{n}}:[t=1] \subseteq T,\|t\| \leq N\right\}$. For every $t \in \mathcal{T}$, set $B_{t}=\{x \in$ $\left.A:\left.f(x)\right|_{m_{n}}=t\right\}$. If there is a $t \in \mathcal{T}$ such that $B_{t}$ is of second category in $\mathcal{F}_{S}(1)$ and $\left\|\cup B_{t}\right\|_{n} \geq n-2$, then we are done.

So suppose no such $t \in \mathcal{T}$ exists. Set

$$
C=\bigcup\left\{B_{t}: t \in \mathcal{T}, B_{t} \text { is of second category in } \mathcal{F}_{S}(1)\right\} .
$$

Then by (5.4), using $|\mathcal{T}| \leq 2^{m_{n}}$,

$$
\|\bigcup C\|_{n} \leq \sup _{t \in \mathcal{T}}\left\|\bigcup B_{t}\right\|+\frac{\log \left(2^{m_{n}}\right)}{m_{n}+1}<n-2+\frac{\log \left(2^{m_{n}}\right)}{m_{n}+1}<n-1 .
$$

However, since $A$ is of second category everywhere in $N_{s} \cap \mathcal{F}_{S}(1)$, we get $I_{n} \subseteq$ $\bigcup A=\bigcup C$. Thus $\|\bigcup C\|_{n}=\log \left(2^{n \cdot m_{n}}+1\right) /\left(m_{n}+1\right) \geq n-1$. This contradiction completes the proof.

Proof of Proposition 5.29. First let $S, T \in[\omega]^{\omega}$ satisfy $S \subseteq \subseteq^{\star} T$. Set $f: \mathcal{F}_{S} \rightarrow \mathcal{F}_{T}$, $f(x)=x \cap I_{T}\left(x \in \mathcal{F}_{S}\right)$. It is easy to verify that $f$ is a Tukey map. 
Now let $S, T \in[\omega]^{\omega}$ satisfy $S \mathbb{}^{\star} T$. As we have seen, $\mathcal{F}_{S \backslash T} \leq_{T} \mathcal{F}_{S}$. So it is enough to prove $\mathcal{F}_{S \backslash T} \mathbb{Z}_{T} \mathcal{F}_{T}$; that is, we can assume $S \cap T=\emptyset$.

Let $f: \mathcal{F}_{S} \rightarrow \mathcal{F}_{T}$ be an arbitrary function. We find $N<\omega$ and $n_{i}<\omega$, $s_{i}, t_{i} \in 2^{<\omega}, A_{i} \subseteq \mathcal{F}_{S}(1)(i<\omega)$ such that

(1) $n_{i} \in S, n_{i}<n_{i+1}, s_{i} \in 2^{m_{n_{i}}}(i<\omega)$;

(2) $t_{i} \in 2^{m_{n_{i}}},\left\|t_{i}\right\| \leq N, t_{i} \sqsubseteq t_{i+1},\left[t_{i}=1\right] \subseteq T(i<\omega)$;

(3) $N_{s_{i}} \cap \mathcal{F}_{S}(1) \neq \emptyset$ and $A_{i}$ is of second category everywhere in $N_{s_{i}} \cap \mathcal{F}_{S}(1)$ $(i<\omega)$;

(4) for every $i<w, f\left[A_{i}\right] \subseteq \mathcal{F}_{T}(N),\left\|\bigcup A_{i+1}\right\|_{n_{i}} \geq n_{i}-2$ and $\left.f(x)\right|_{m_{n_{i}}}=t_{i}$ $\left(x \in A_{i+1}\right)$.

Suppose first the construction is done; we show that $f$ is not a Tukey map. For every $i<\omega$, let $X_{i} \subseteq A_{i+1}$ be minimal such that $\left(\bigcup X_{i}\right) \cap I_{n_{i}}=\left(\bigcup A_{i+1}\right) \cap I_{n_{i}}$. Then $\left|X_{i}\right| \leq b_{n_{i}}(i<\omega)$. By $4, x=\bigcup_{i<\omega} \bigcup X_{i}$ satisfies $\|x\|_{n_{i}} \geq n_{i}-2(i<\omega)$, i.e. $\bigcup_{i<\omega} X_{i}$ is unbounded in $\mathcal{F}_{S}$.

We show that $\bigcup_{i<\omega}\left\{f(x): x \in X_{i}\right\} \subseteq \mathcal{F}_{T}$ is bounded. Set $\tau=\bigcup_{i<\omega} t_{i}$; then by 2. $\tau \in \mathcal{F}_{T}$ and $\|\tau\| \leq N$. For every $n \in T$, by (5.4), 4 and $n \notin S$,

$$
\begin{aligned}
& \left\|\bigcup \bigcup_{i<\omega}\left\{f(x): x \in X_{i}\right\}\right\|_{n} \leq\left\|\tau \cup \bigcup\left\{f(x): x \in X_{i}, n_{i}<n\right\}\right\|_{n} \\
& \leq N+\frac{\log \left(1+\sum\left\{b_{n_{i}}: n_{i}<n\right\}+1\right)}{m_{n}+1} \leq N+\frac{\log \left(m_{n}+2\right)}{m_{n}+1} \leq N+1,
\end{aligned}
$$

so the statement follows.

It remains to perform the construction. We have $f\left[\mathcal{F}_{S}(1)\right] \subseteq \bigcup_{N<\omega} \mathcal{F}_{T}(N)$, so there are $N<\omega$ and $A_{0} \subseteq \mathcal{F}_{S}(1)$ such that $f\left[A_{0}\right] \subseteq \mathcal{F}_{T}(N)$ and $A_{0}$ is of second category in $\mathcal{F}_{S}(1)$. So we can find $n_{0} \in S, s_{0} \in 2^{n_{0}}$ such that $N_{s_{0}} \cap \mathcal{F}_{S}(1) \neq \emptyset$ and $A_{0}$ is of second category everywhere in $N_{s_{0}} \cap \mathcal{F}_{S}(1)$.

Let $i<\omega$ be arbitrary and suppose that $n_{i}, s_{i}$, and $A_{i}$ have already been found. We apply Lemma 5.30 with $S, T, N, A=A_{i}, n=n_{i}$ and $s=s_{i}$. We get a $t_{i} \in 2^{m_{n_{i}}}$ such that $\left[t_{i}=1\right] \subseteq T,\left\|t_{i}\right\| \leq N, A_{i+1}=\left\{x \in A_{i}:\left.f(x)\right|_{m_{n_{i}}}=t_{i}\right\} \subseteq \mathcal{F}_{S}(1)$ is of second category and $\left\|\bigcup A_{i+1}\right\|_{n_{i}} \geq n_{i}-2$. It remains to choose $n_{i+1} \in S, n_{i+1}>n_{i}$, $s_{i+1} \in 2^{n_{i+1}}$ such that $N_{s_{i+1}} \cap \mathcal{F}_{S}(1) \neq \emptyset$ and $A_{i+1}$ is of second category everywhere in $N_{s_{i+1}} \cap \mathcal{F}_{S}(1)$. Then 11, 3 and 4 hold, and for 2 it remains to show $t_{i-1} \sqsubseteq t_{i}$ if $i>0$. However, this follows from $A_{i+1} \subseteq A_{i}$ using the inductive hypothesis $\left.f(x)\right|_{m_{n_{i-1}}}=t_{i-1}\left(x \in A_{i}\right)$. So the proof is complete.

\section{Problems}

In our present work we did not apply some well-understood methods for studying infinite dimensional perfect set theorems. We close this paper with a survey of possible further research directions and state some related open problems.

6.1. General symmetric topologies, analytic relations. Apart from the usual product topology, there are several topologies on $\mathbb{R}^{\omega}$ which are symmetric (i.e. open sets remain open under arbitrary permutation of coordinates) and which are important in applications, e.g. the box topology or the topologies induced by the $\ell^{p}(1 \leq p \leq \infty)$ norms. There is no reason to believe that the product topology is the most appropriate for the formulation of optimal infinite dimensional perfect 
set theorems. It would be interesting to find weaker largeness assumptions in these finer topologies than our $X^{\omega} \backslash A \in \mathbb{M}$ in Theorem 4.1, which still imply the existence of a non-empty perfect $A$-homogeneous set. E.g. it is easy to construct a symmetric dense open set $A \subseteq \mathbb{R}^{\omega}$ such that there is no infinite $A$-homogeneous set. But in $\mathbb{R}^{\omega}$ endowed with the box topology, for every symmetric dense open set there is an infinite homogeneous set. Compare this with the remarks following Corollary 2.3.

Note also that ironically, all of our perfect set theorems hold for co-analytic relations, while the rank approach of [13] and 23] is able to handle analytic relations. Observe that to every construction using finite approximations, one can associate the tree of finite approximations ordered by end-extension such that the ill-foundedness of this tree is equivalent with the existence of the limit object of the construction. Therefore the infinite dimensional counterpart of [13, Proposition 4.1, p. 151] is natural to formulate and easy to prove. The more involved task would be to study the existence of universal relations as in [13, Section 5] and to characterize the resulting rank as in 23. It seems that these investigations can be carried out for any of the above mentioned refinements of the product topology, as well.

6.2. More fusion games. It seems informative to study fusions of other games of descriptive set theory, especially those of Separation games and Wadge games. We remark that the way we increased the dimension in our fusion scheme was completely arbitrary; different schemes characterize other notions of smallness.

We propose an explicit modification of $\mathcal{G}_{\omega}$ of Definition 3.2 which seems particularly interesting to study. Consider the game $\mathcal{G}_{\omega}^{\mathrm{m}} \subseteq \mathcal{G}_{\omega}$, where, in addition, in the $(n+1)^{\text {th }}$ move player $I$ is required to play $\left(U_{i}(2(n+1-i))\right)_{i \leq n+1}$ such that $U_{n+1}(0) \subseteq \bigcup_{i \leq n} U_{i}(2(n-i)+1)$; otherwise the game is unchanged. For player $I I$ this game is easier to win, still one can show that the existence of a winning strategy for player $I I$ in $\mathcal{G}_{\omega}^{\mathrm{m}}(A)$ implies the existence of a non-empty perfect $A$-homogeneous set.

Unfortunately we could not characterize the existence of a winning strategy for player $I$ in $\mathcal{G}_{\omega}^{\mathrm{m}}(A)$. Nevertheless, we expect that fusion games modified in such ways can provide sharper results.

6.3. Other Choquet topologies and forcings. The Ellentuck topology and the density topologies are Choquet, in particular Theorem 4.1 can be applied to them. However, they fail the Kuratowski-Ulam property, which is crucial for Lemma 4.4 and so for all the results of Section 4.1 and Section 4.2, E.g. our methods do not allow us to prove the counterparts of Theorem 1.2 and Theorem 4.10, in which "meager" is replaced by "Lebesgue null" and "Cohen" is replaced by "random" 1

Problem 6.1. ${ }^{1}$ Let $A \subseteq \mathbb{R}^{\omega}$ be a co-analytic set. Does the existence of an $A$ homogeneous set of positive outer Lebesgue measure imply the existence of a nonempty perfect $A$-homogeneous set?

Problem 6.2. ${ }^{1}$ Let $V$ be a model obtained from a model of the Continuum Hypothesis by adding $\omega_{2}$ random reals. In $V$, let $A \subseteq \mathbb{R}^{\omega}$ be a co-analytic set. Does the existence of an $A$-homogeneous set of cardinality $\omega_{2}$ imply the existence of a non-empty perfect $A$-homogeneous set?

\footnotetext{
${ }^{1}$ Recently J. Zapletal 31 showed that these analogous results hold, and he gave affirmative answers to Problem 6.1 and Problem 6.2
} 
Note that the finite dimensional analogue of Problem 6.1 holds for every Lebesgue measurable set $A$ by the measure version of Mycielski's Theorem (see e.g. 28, Theorem 6.40]), while the finite dimensional analogue of Problem 6.2 holds for every analytic set $A$ by $[23$, Fact 1.16, p. 23].

These problems may be related to the following.

Problem 6.3. Let $A \subseteq 2^{\omega} \times 2^{\omega}$ be a co-meager set. Is it true in ZFC that there is an $A$-homogeneous set which is of second category everywhere?

If e.g. $\operatorname{cof}(\mathcal{M})=\operatorname{cov}(\mathcal{M})$, then by an easy transfinite argument, for every comeager set $A \subseteq 2^{\omega} \times 2^{\omega}$ one can construct an $A$-homogeneous set which is of second category everywhere. Also note the counterpart of Problem 6.3 involving Lebesgue measure fails.

Theorem 6.4 ([2], 7]). Let $V$ be a model obtained from a model of the Continuum Hypothesis by adding $\omega_{2}$ Cohen reals. Then in $V$, there is an $F_{\sigma}$ set $A \subseteq 2^{\omega} \times 2^{\omega}$ of Lebesgue measure 1 such that there exists no A-homogeneous set of positive outer Lebesgue measure.

The proof of Theorem 6.4 is based on the observation that in Cohen extensions, if for an $F_{\sigma}$ set $A \subseteq 2^{\omega} \times 2^{\omega}$ there is an $A$-homogeneous set of positive outer Lebesgue measure, then there is an $A$-homogeneous set of positive Lebesgue measure. Thus it is likely that Problem 6.1 has an affirmative answer in Cohen extensions. However, the proof of Theorem 6.4 does not have a straightforward modification valid for random extensions, so we could not obtain a negative answer to Problem 6.3.

Problem 6.3 was motivated by the question of whether the largeness assumption in Theorem 1.2 is the natural analogue of the largeness assumption of Mycielski's Theorem. As we pointed out above, this is consistently true, since for sets $A \subseteq$ $2^{\omega} \times 2^{\omega}$ having the Baire property, being co-meager and having an $A$-homogeneous set which is of second category everywhere are consistently equivalent.

In Cohen extensions there are perfectly meager sets of cardinality $2^{\aleph_{0}}$, so the largeness assumption in Theorem 1.2 is consistently not optimal. It would be interesting to know whether the converse is also consistent; the following is also related to Problem 6.3

Problem 6.5. Is it consistent with ZFC that $H \subseteq \mathbb{R}$ is perfectly meager if and only if there is an open set $A \subseteq \mathbb{R}^{\omega}$ such that $I S_{\omega}(H) \subseteq A$ but there is no non-empty perfect $A$-homogeneous set?

6.4. Local results. In [15] the following "local" infinite dimensional perfect set theorem was obtained (see also [28, Corollary 6.48]). We call a sequence $\left(x_{n}\right)_{n<\omega} \in$ $\mathbb{R}^{\omega}$ rapidly increasing if $0<x_{n+2}-x_{n+1}<x_{n+1}-x_{n}(n<\omega)$. The set of rapidly increasing sequences is denoted by $\mathcal{R}$.

Theorem 6.6 ([15, Theorem 2, p. 275]). For every finite Baire measurable coloring of $\mathcal{R}$ there is a perfect set $P \subseteq \mathbb{R}$ such that $P^{\omega} \cap \mathcal{R}$ is monochromatic.

Ramsey-type theorems such as Theorem 6.6 are very important, e.g. Theorem 5.14 is also based on Theorem 6.6. The most general results of this nature are known as the Halpern-Läuchli Theorems (see e.g. [10] or 28] and the references therein). It is easy to see that there is an open tower $\mathbb{U}$ such that $[\mathbb{U}]=\mathcal{R}$, in particular $\mathcal{R} \notin \mathbb{M}$. It would be very useful to explore the possible interplay between our approach and such local results. 
6.5. Cofinal types of analytic ideals. A deeper analysis of the construction in the proof of Theorem 5.21 reveals it uses that $E$ of Definition 5.23 is an equivalence relation in an essential way. Thus this method cannot yield a Borel ideal satisfying the conditions of Theorem [5.21, On the other hand, the construction in the proof of Theorem 2.1 is the base step of the construction of an $F_{\sigma}$ ideal satisfying the conditions of Theorem 5.21. Therefore we expect a positive answer to the following problems.

Problem 6.7. Is there an $F_{\sigma}$ ideal $\mathcal{I} \subseteq \mathcal{P}(\omega)$ such that $\left[\omega_{1}\right]^{<\omega} \leq_{T} \mathcal{I}$ but $\mathcal{I}$ has no non-empty perfect strongly unbounded subsets?

Problem 6.8 ([16, Conjecture 1, p. 194]). Is there an $F_{\sigma}$ ideal $\mathcal{I} \subseteq \mathcal{P}(\omega)$ such that $\left[\omega_{1}\right]^{<\omega} \leq_{T} \mathcal{I}$ but $\omega^{\omega} \underline{\leq}_{T} \mathcal{I}$ ?

We also expect the consistency of the failure of the primality property for $\mathcal{I}_{\max }$.

Problem 6.9. Is it consistent with $\mathrm{ZFC}$ that $\mathcal{I}_{\max }$ fails the primality property, i.e. there exist analytic ideals $\mathcal{I}, \mathcal{J} \subseteq \mathcal{P}(\omega)$ such that $\mathcal{I}_{\max } \leq_{T} \mathcal{I} \oplus \mathcal{J}$ but $\mathcal{I}_{\max } \underline{\leq}_{T} \mathcal{I}$ and $\mathcal{I}_{\max } \not_{T} \mathcal{J}$ ?

At the present stage of research, one could wonder whether for every analytic ideal $\mathcal{I} \subseteq \mathcal{P}(\omega),\left[\omega_{2}\right]^{<\omega} \leq_{T} \mathcal{I}$ implies that $\mathcal{I}$ has a non-empty perfect strongly unbounded subset. However, we expect that the affirmative answer to Problem 6.7 will be based on a construction which is flexible enough to rule out such speculations.

\section{REFERENCES}

1. T. Bartoszyński, Invariants of Measure and Category, Handbook of set theory. Vols. 1, 2, 3, 491-555, Springer, Dordrecht, 2010. MR2768686

2. M. R. Burke, A theorem of Friedman on rectangle inclusion and its consequences, note.

3. D. Cenzer, R. D. Mauldin, On the Borel class of the derived set operator, Bull. Soc. Math. France 110 (1982), no. 4, 357-380. MR694756 (85b:54058)

4. D. Cenzer, R. D. Mauldin, On the Borel class of the derived set operator II, Bull. Soc. Math. France 111 (1983), no. 4, 367-372. MR763550 (86a:54046)

5. D. H. Fremlin, The partially ordered sets of measure theory and Tukey's ordering. Dedicated to the memory of Professor Gottfried Köthe, Note Mat. 11 (1991), 177-214. MR.1258546 (95e:06006)

6. D. H. Fremlin, Families of compact sets and Tukey's ordering, Atti Sem. Mat. Fis. Univ. Modena 39 (1991), no. 1, 29-50. MR.1111757 (92c:54032)

7. H. Friedman, Rectangle inclusion problems, note.

8. L. Harrington, S. Shelah, Counting equivalence classes for co- $\kappa$-Souslin equivalence relations, Logic Colloquium '80 (Prague, 1980), 147-152, Stud. Logic Foundations Math., 108, NorthHolland, Amsterdam-New York, 1982. MR673790 (84c:03088)

9. T. Jech, Set Theory (third edition), Springer, 2003. MR.1940513 (2004g:03071)

10. V. Kanellopoulos, Ramsey families of subtrees of the dyadic tree, Trans. Amer. Math. Soc. 357 (2005), no. 10, 3865-3886. MR2159691 (2006d:05052)

11. A. S. Kechris, Classical Descriptive Set Theory, Graduate Texts in Mathematics 156, SpringerVerlag, 1994. MR1321597 (96e:03057)

12. W. Kubiś, Perfect cliques and $G_{\delta}$ colorings of Polish spaces, Proc. Amer. Math. Soc. 131 (2003), no. 2, 619-623. MR1933354 (2004g:54043)

13. W. Kubiś, S. Shelah, Analytic colorings, Ann. Pure Appl. Logic 121 (2003), no. 2-3, 145-161. MR.1982945 (2004j:03055)

14. K. Kunen, Set theory. An introduction to independence proofs, Studies in Logic and the Foundations of Mathematics 102, North-Holland Publishing Co., Amsterdam-New York, 1980. MR597342 (82f:03001)

15. A. Louveau, S. Shelah, B. Veličković, Borel partitions of infinite subtrees of a perfect tree, Ann. Pure Appl. Logic 63 (1993), no. 3, 271-281. MR1237234 (94g:04003) 
16. A. Louveau, B. Veličković, Analytic ideals and cofinal types, Ann. Pure Appl. Logic 99 (1999), no. 1-3, 171-195. MR1708151 (2000g:03111)

17. K. Mazur, A modification of Louveau and Veličković's construction for $F_{\sigma}$-ideals, Proc. Amer. Math. Soc. 128 (2000), no. 5, 1475-1479. MR1626442 (2000j:03067)

18. A. W. Miller, Mapping a set of reals onto the reals, J. Symbolic Logic, Vol. 48 (1983), No. 3, 575-584. MR716618 (84k:03125)

19. A. W. Miller, On the length of Borel hierarchies, Ann. Math. Logic 16 (1979), no. 3, 233-267. MR:548475 (80m:04003)

20. J. Mycielski, Independent sets in topological algebras, Fund. Math. 55 (1964), 139-147. MR0173645 (30:3855)

21. M. Sabok, Forcing, games and families of closed sets, Trans. Amer. Math. Soc., to appear.

22. M. Sabok, On Idealized Forcing, Instytut Matematyczny Uniwersytetu Wrocławskiego, Ph.D. thesis.

23. S. Shelah, Borel sets with large squares, Fund. Math. 159 (1999), 1-50. MR1669643 (2000i:03073)

24. S. Solecki, Covering analytic sets by families of closed sets, J. Symbolic Logic 59 (1994), no. 3, 1022-1031. MR.1295987 (95g:54033)

25. S. Solecki, S. Todorčević, Cofinal types of topological directed orders, Ann. Inst. Fourier (Grenoble) 54 (2004), no. 6, 1877-1911 (2005). MR2134228 (2006k:03089)

26. S. Todorčević, A classification of transitive relations on $\omega_{1}$, Proc. London Math. Soc. (3) 73 (1996), no. 3, 501-533. MR.1407459 (97k:04001)

27. S. Todorčević, Directed sets and cofinal types, Trans. Amer. Math. Soc. 290 (1985), no. 2, 711-723. MR792822 (87a:03084)

28. S. Todorčević, Introduction to Ramsey Spaces, Annals of Mathematics Studies, Vol 174, Princeton University Press, 2010. MR2603812

29. J. W. Tukey, Convergence and uniformity in topology, Annals of Mathematics Studies, no. 2. Princeton University Press, Princeton, N.J., 1940. MR0002515(2:67a)

30. J. Zapletal, Forcing idealized, Cambridge Tracts in Mathematics 174, Cambridge University Press, Cambridge, 2008. MR2391923 (2009b:03002)

31. J. Zapletal, Homogeneous sets of positive outer measure, preprint.

Department of Mathematics, University of Toronto, 40 St. George Street, Toronto, ONTARIO, CANADA

E-mail address: matrait@renyi.hu

Current address: Alfréd Rényi Institute of Mathematics, Hungarian Academy of Sciences, 13-15 Reáltanoda Street, Budapest H-1053 Hungary 